University of Texas Rio Grande Valley

ScholarWorks @ UTRGV

Physics and Astronomy Faculty Publications

and Presentations

College of Sciences

$5-6-2021$

Study of efficient methods of detection and reconstruction of

gravitational waves from nonrotating 3D general relativistic core collapse supernovae explosion using multilayer signal estimation method

Soma Mukherjee

The University of Texas Rio Grande Valley

Gaukhar Nurbek

The University of Texas Rio Grande Valley

Oscar Valdez

The University of Texas Rio Grande Valley

Follow this and additional works at: https://scholarworks.utrgv.edu/pa_fac

Part of the Astrophysics and Astronomy Commons, and the Physics Commons

Recommended Citation

Mukherjee, Soma, Gaukhar Nurbek, and Oscar Valdez. 2021. "Study of Efficient Methods of Detection and Reconstruction of Gravitational Waves from Nonrotating 3D General Relativistic Core Collapse Supernovae Explosion Using Multilayer Signal Estimation Method." Physical Review D 103 (10): 103008. https://doi.org/10.1103/PhysRevD.103.103008.

This Article is brought to you for free and open access by the College of Sciences at ScholarWorks @ UTRGV. It has been accepted for inclusion in Physics and Astronomy Faculty Publications and Presentations by an authorized administrator of ScholarWorks @ UTRGV. For more information, please contact justin.white@utrgv.edu, william.flores01@utrgv.edu. 


\title{
Study of efficient methods of detection and reconstruction of gravitational waves from nonrotating 3D general relativistic core collapse supernovae explosion using multilayer signal estimation method
}

\author{
Soma Mukherjee®, Gaukhar Nurbek®, and Oscar Valdez \\ Department of Physics and Astronomy, University of Texas Rio Grande Valley, \\ One W University Boulevard, Brownsville, Texas 78520, USA
}

(Received 13 December 2020; accepted 31 March 2021; published 6 May 2021)

\begin{abstract}
In the post-detection era of gravitational wave ( $\mathrm{GW}$ ) astronomy, core collapse supernovae (CCSN) are one of the most interesting potential sources of signals arriving at the Advanced LIGO detectors. Mukherjee et al. have developed and implemented a new method to search for GW signals from the CCSN search based on a multistage, high accuracy spectral estimation to effectively achieve higher detection signal to noise ratio (SNR). The study has been further enhanced by incorporation of a convolutional neural network (CNN) to significantly reduce false alarm rates (FAR). The combined pipeline is termed multilayer signal estimation (MuLaSE) that works in an integrative manner with the coherent wave burst (cWB) pipeline. In order to compare the performance of this new search pipeline, termed "MuLaSECC", with the cWB, an extensive analysis has been performed with two families of core collapse supernova waveforms corresponding to two different three dimensional (3D) general relativistic CCSN explosion models, viz. Kuroda 2017 and the Ott 2013. The performance of this pipeline has been characterized through receiver operating characteristics (ROC) and the reconstruction of the detected signals. The MuLaSECC is found to have higher efficiency in low false alarm range, a higher detection probability of weak signals and an improved reconstruction, especially in the lower frequency domain.
\end{abstract}

DOI: 10.1103/PhysRevD.103.103008

\section{INTRODUCTION}

The first direct detection of gravitational waves (GW) happened in 2015 when the two LIGO detectors [1] at Hanford and Livingston detected the GW signal from a binary black hole (BBH) system [2]. Since then, several $\mathrm{BBH}$ signals have been detected by the LIGO detectors. A watershed moment happened in 2017 when the LIGO and Virgo [3] detectors detected GW signal from a colliding neutron star system (BNS) [4]. Yet another landmark discovery took place in 2019 scientists from LIGO and Virgo have announced the discovery of an object of 2.6 solar masses, placing it firmly in the "mass gap," as it merged with a black hole of 23 solar masses [5].

In the post-detection era of GW astronomy, core collapse supernovae (CCSN) are one of the most interesting potential sources of signals arriving at the Advanced LIGO (aLIGO [6]) detectors. CCSN are rare, but the associated gravitational radiation is likely to carry profuse information about the underlying processes driving the supernovae. Studies have shown [7] that the sources with neutrino-driven explosions are detectable at the distances approaching $5 \mathrm{kpc}$. For magneto-rotationally driven explosions the distances are up to $54 \mathrm{kpc}$. Waveforms for extreme emission models are detectable up to $28 \mathrm{Mpc}$. More details about the detection ranges corresponding to different types of explosion models are discussed in Sec. II. Since the signals from these sources are weak, methods that can improve the sensitivity of searches for GW signals from CCSN are desirable, especially in the advanced detector era. Several methods have been proposed [8-10] based on various likelihood-based regulators that work on data from a network of detectors to detect burst signals (as is the case for signals from CCSN) from potential GW sources. To address this problem, Mukherjee et al. [11] have developed and implemented a new technique of signal estimation and enhancement in the CCSN search pipeline based on a multistage, high accuracy spectral estimation to effectively achieve higher signal to noise ratio (SNR) [12-15]. In the current study the methods has been further enhanced by incorporation of a convolutional neural network $(\mathrm{CNN})$ [16-20] to reduce false alarm significantly. The combined pipeline is termed Multi-Level Signal Estimation (MuLaSE). The MuLaSE works in combination with LIGO's coherent wave burst (cWB) [21] pipeline, but the MuLaSE component enhances the performance of the search method, as well as provides more effective parameter estimation of the detected signal. The results have been generated using the CCSN waveforms described by Kuroda et al. [22,23] and Ott et al. [24]. The former results are derived from relativistic three-dimensional (3D) CCSN simulations of a nonrotating 11.2, 15 and 40 solar mass 
star using three different nuclear equations of state $(\mathrm{EoS})$. In this study, we have used the waveform that was generated by using the 11.2 solar mass progenitor. The duration of the simulation is about $350 \mathrm{~ms}$ after bounce, where the development of the standing accretion shock instability (SASI) is shown to be dependent on stiffness of nuclear EoS. The generated waveform displays a new structure in which the typical GW frequency increases with time due to an accumulating accretion to the protoneutron star (PNS). The newly observed quasiperiodic signal appears in the frequency range from 100 to $200 \mathrm{~Hz}$ and persists for $150 \mathrm{~ms}$ before neutrino-driven convection dominates over the SASI. The GW signal generated in this study is within the detection limits of LIGO, advanced Virgo, and KAGRA [25] for Galactic events. The latter [24] describes a 3D general relativistic simulation of the post-core-bounce phase of the collapse of a 27 solar mass star including the development of SASI and neutrinodriven convection. The waveform is expected to serve as an observational probe of the post-bounce dynamics and may determine the primary hydrodynamic instability when combined with the emitted neutrino information.

The paper is organized as follows. Section II gives an overview of the work done on GW detection effort for the core collapse supernovae (CCSN) sources and also a description of the models used in this study. Section III summarizes the MuLaSE algorithm and the search pipeline. Section IV discusses the analysis results and Sec. V gives conclusions and future directions.

\section{A REVIEW OF PREVIOUS WORK}

Work done in the domain of GW from CCSN can be broadly grouped into two categories. These are

(i) theoretical simulation of CCSN explosion models and

(ii) methods of detection of GW from CCSN.

In case of (i), a wide variety of CCSN explosion scenarios has been discussed in the literature. Currently a large repertoire of CCSN waveforms is available. These waveforms vary in terms of explosion models and parameters. With more advanced and faster computing infrastructure, more extensive simulations involving a wide net of complexities has been made possible. This review of waveform simulations is not exhaustive, we will describe some recently developed ones to provide a broad idea about the direction in this field and also describe the waveforms we have chosen to use in this paper. More extensive information can be sought at [26]

Mezzacappa et al. [27] report on 3D simulation of GW signal of a 15 solar mass star using the neutrino hydrodynamics code CHIMERA [28]. Both time and spectral signatures of the emitted GW have been calculated. The main signatures in their waveform include an emission below $200 \mathrm{~Hz}$ that result from neutrino-driven convection and SASI, and a high-frequency emission $(\geq 600 \mathrm{~Hz})$ from the PNS convective layer. This study indicates that the GW spectrum in the range $20-1000 \mathrm{~Hz}$ will be accessible for a galactic event. Powell and Muller [29] have conducted a recent study using 3D simulations of the core-collapse of massive rotating and nonrotating progenitors performed using the general relativistic neutrino hydrodynamics code COCONUT-FMT [30]. The progenitor models include 39 and 20 solar masses and an 18 solar mass super giant. The 39 solar mass model is a rapid rotator, whereas the two other progenitors are nonrotating. Their study has shown that the neutrino-driven explosions are successful but the red supergiant model fails to explode. It has been found that the rotation significantly changed the dependence of the characteristic GW frequency of the f-mode on the PNS parameters. It was predicted that the GW emission from the CCSN could be detected up to $2 \mathrm{Mpc}$ by the Einstein Telescope [31]

In the current study we have analyzed two 3D general relativistic CCSN explosion models [22,24] using the MuLaSE pipeline. Both these waveforms have been chosen within the LIGO Scientific Collaboration [32] to be used in the analysis of the third observing run (O3) of LIGO.

Kuroda et al. $[22,23]$ have presented general relativistic 3D simulations with three different nuclear EoS. In this simulation, a strong SASI development was observed for softer EoS. The evolution shows the first prompt convection phase, the sloshing-SASI phase, which shifts to the spiral mode and finally to the neutrino-driven convection phase. The study confirmed previously reported emissions originated from the PNS surface g-mode oscillation $[33,34])$. In the softest EoS model, in addition to a strong SASI, a new low-frequency quasi-periodic emission was observed between 100-200 Hz. This emission was spatially localized between $10-20 \mathrm{~km}$. Further analysis of the SASI modes, the mass accretion rate at $20 \mathrm{~km}$ and of the quadrupole mode of the central core deformation, seem to indicate that the temporally perturbed mass accretion in association with the SASI may pierce into the PNS surface to excite the oscillation between $10-20 \mathrm{~km}$, which is responsible for the GW emission. Andresen et al. [35] have also reported a similar result at low-frequency GW. The predicted waveforms are well within the detectable frequency range of Advanced LIGO, Virgo and KAGRA detectors. The peak frequency appears at 635, 671, and $681 \mathrm{~Hz}$ in order of the stiffness of nuclear EoS. At this frequency range, the SNR reaches 10 at $10 \mathrm{kpc}$. As for the newly observed low frequency emission at peak frequency $129 \mathrm{~Hz}$, the SNR reaches an approximate value of 50. It is thus expected that such events will be visible with the current network of detectors. The current study has been performed with the s11 waveform.

Ott et al. [24] have carried out four 3D relativistic core collapse and post-bounce simulations of the 27 solar mass stars by systematically varying the rate of neutrino energy deposition. These simulations were free of imposition of articial inner boundary conditions or of symmetry 
assumptions or approximations for the gravitational field. Ott et al.'s models show a strong growth of convective instability which is driven by the negative entropy gradient left behind by the stalling shock. Subsequently, convection is driven by neutrino energy deposition in the gain layer. The SASI remains a sub-dominant instability in all models in this study. In addition, the SASI has been found to be strongest in the model with the least neutrino heating and the weakest neutrino-driven convection.

Much work has been done to address the data analysis issues associated with detection and parameter estimation of the signals. In 2016, Abbott et al. [36] published results from a search for gravitational-wave bursts coincident with two CCSN observed optically in 2007 and 2011 using LIGO, Virgo and GEO600 [37] data. The targeted CCSN were selected within approximately $15 \mathrm{Mpc}$. Coincident operation of at least two interferometers at the time of core collapse were considered. Although no GW sinals were found, this study laid the foundation of targeted searches with optical signatures of CCSN. A further study by the same authors [7] include possibility of detection of GW from CCSN within a source distance of approximately $20 \mathrm{Mpc}$. The sources with neutrino-driven explosions are detectable at the distances approaching $5 \mathrm{kpc}$ For magnetorotationally driven explosions the distances are up to $54 \mathrm{kpc}$. However, waveforms for extreme emission models are detectable up to $28 \mathrm{Mpc}$. The GW energy was constrained at the levels of $4.27 \times 10^{-4} M_{\text {sun }} c^{2}$ and $0.128 \times$ $M_{\text {sun }} c^{2}$ for emissions at 235 and $1304 \mathrm{~Hz}$, respectively. These constraints are 2 orders of magnitude more stringent than previously derived. In 2018, Hayama et al. [38] analyzed the circular polarization of GW using results from a 3D analysis of CCSN from a 15 solar mass nonrotating progenitor. The detection analysis was performed by using a coherent network of detectors viz, LIGO, Virgo and KAGRA. Their result demonstrated that the SASI-induced accretion owes to the PNS create a lowfrequency modulation in the 100 and $200 \mathrm{~Hz}$ range. By estimating the SNR of the GW polarization, it was shown that the detection horizon of the circular polarization extends significantly farther. Gossan et al. [39] have shown that GWs from CCSN within $5 \mathrm{Mpc}$ are detectable, using realistic noise at the predicted sensitivity of the Advanced LIGO and Advanced Virgo detectors. Their study has considered extreme $\mathrm{GW}$ emission scenarios for more distant CCSN. A three-detector network in such a scenario is predicted to detect neutrino-driven CCSN explosions out to $\approx 5.5 \mathrm{kpc}$, while rapidly rotating core collapse will be detectable out to the Large Magellanic Cloud at $50 \mathrm{kpc}$. Using phenomenological waveforms [40,41], e.g., longlived bar-mode instabilities and disk fragmentation instabilities, Gossan et al.'s study, have been shown to detect emission from all considered long-lived bar-mode instability waveforms in the direction of M31 up to $0.77 \mathrm{Mpc}$ with 2019 LIGO three detector network. In addition, for
CCSN in the direction of M82, it has been seen that the emission from long-lived bar-mode instabilities will be detectable out to $\approx 9 \mathrm{Mpc}$ with the same network. Bizouard et al. [42] have shown a parameter estimation approach for GW associated with PNS oscillation. The study involves a set of 1D CCSN simulations to model evolution of the PNS properties as a function of frequency of the dominant g-mode to infer about the time evolution with a combination of the mass and the radius of the PNS. PNS properties for a galactic source using the current detectors is shown to be quite feasible.

In recent times, there is an effort to use machine learning (ML) in CCSN analysis with a eye to reduce false alarm (FA). Cavaglia et al. [43] have studied an ML-based method to discriminate galactic GW CCSN signals from glitches, which in turn reduces FA and improves detection statistic. In another study, Iess et al. [44] describe a search and classification procedure for $\mathrm{GW}$ emitted by CCSN, using CNN combined with an event trigger generator known as wavelet detection filter (WDF). The authors used both a 1-D CNN search using time series of GW strain data, and a 2-D CNN search using time-frequency images. Using design sensitivities of the current and future detectors, their study indicated a classification accuracy of over 95\% for both 1-D and 2-D CNN pipelines. Chan et al. [45] also demonstrated the application of $\mathrm{CNN}$ to distinguish glitches from CCSN signals. Using simulated time series of GW detectors, they have shown that a network of Advanced LIGO, Advanced VIRGO and KAGRA (HLVK) is likely to detect a magneto-rotational CCSN out to the large or even small Magellanic clouds. A neutrino-driven event is likely to be detectable within 5 and $3 \mathrm{kpc}$ respectively. George et al. [46] used a data set of twenty-two classes of glitches from LIGO's observation run, and demonstrated that CNN significantly reduced the training time and achieved accuracy above $98.8 \%$. Astone et al. [47] have analyzed data from the LIGO, Virgo and KAGRA network with a CNN-based classication procedure of the time-frequency images using phenomenological waveforms in Gaussian noise. Lopez-Portilla [48] et al. have used $\mathrm{O} 2$ data to demonstrate a newly developed MiniInception Resnet neural network using time-frequency images of simulated phenomenological signals. The authors have computed the detection eciency versus the source distance. Their results indicate a detection efficiency of $70 \%$ at a false alarm rate (FAR) lower than 5\%. The current study is the first that incorporates $\mathrm{CNN}$ as an integral part of search pipeline which also comprises a sophisticated new element of output SNR enhancement [11] and will henceforth be termed "MuLaSECc" to represent multilayer signal enhancement with CNN and cWB.

\section{SEARCH ALGORITHM AND PIPELINE}

The pipeline is based on the multilayer signal enhancement (termed "MuLaSE") algorithm that has been 


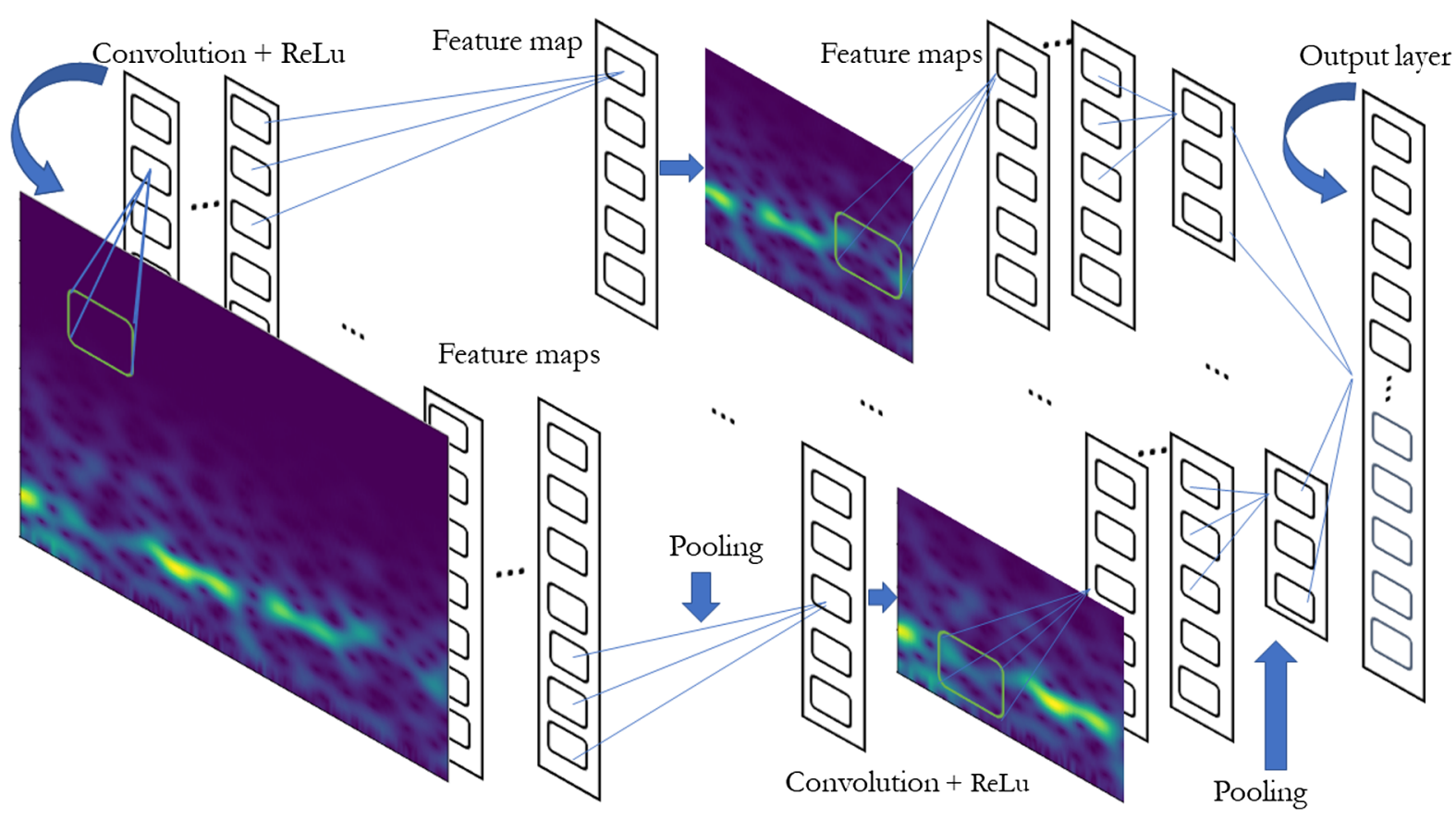

FIG. 1. This figure shows the main steps involved in a typical CNN. A CNN convolves learned features with input data and uses 2D convolutional layers, making it suitable for processing 2D images. CNN has an input layer, and output layer, and hidden layers. The hidden layers usually consist of convolutional layers that pass information to following layers, ReLU layers, pooling layers that combine the outputs of clusters of neurons into a single neuron, and fully connected layers that connect every neuron in one layer to the next level.

described in great detail in [11] and hence We will not repeat the derivation in this paper. A reader can refer to the previous papers for full information about the method. Similarly, the cWB algorithm has been described in detail in [21] and thus we would avoid repetition in this paper.

The CNN [16-20] has been used in a wide range of classification problems for several decades now. CNN is very fast and effective in image classification problems [49]. The CNN is a class of deep learning [50] neural networks. Image classification is the process of taking an input (like a picture) and giving an output or a probability that the input belongs to a particular class of objects. In the current study, this is provided as time-frequency plots of glitches or signals detected in the MuLaSECC pipeline. A CNN convolves learned features with input data and uses 2D convolutional layers, making it suitable for processing 2D images. CNN has an input layer, and output layer, and hidden layers. The hidden layers usually consist of convolutional layers that pass information to following layers, rectified linear unit (ReLU) layers, pooling layers that combine the outputs of clusters of neurons into a single neuron, and fully connected layers that connect every neuron in one layer to the next level. A reader may see [51] for an excellent overview.

A CNN works by extracting features from images that are learned while the network trains on a set of images. Hidden layers of a CNN increase the complexity of the learned features. A CNN follows the sequential steps given below in a repetitive manner until a well-trained network is obtained. Figure 1 gives a schematic diagram of how the feature elements are created from a time-frequency image. The process has the following steps.

(i) starts with time-frequency input image;

(ii) creates a feature map by applying filters to the input time-frequency image;

(iii) applies a ReLU function to increase nonlinearity;

(iv) applies a pooling layer to each feature map and creates one single vector of pooled images;

(v) inputs the vector into a fully connected artificial neural network;

(vi) processes the features through the network. The final fully connected layer provides the voting of the classes that were after;

(vii) trains through forward propagation and back propagation for many, many epochs.

In general, for a color image (as is in our case), a 3D array of pixels with a blue layer, a green layer, and a red layer is produced. The main purpose of the convolution step is to extract features from the input image. This, starting with an input image, a feature detector, and a feature map, the application of filter to the input image is achieved through the multiplication of the matrices. In each position, the filter multiplies the values in the filter with the original values in the pixel by element wise multiplication. The multiplications are summed up, creating a single number. The array thus produced is called a feature map or an activation map. The ReLU layer involves applying an activation function onto your feature maps to increase 
nonlinearity (images being nonlinear) in the network. It removes negative values from an activation map by setting them to zero. The same effect can sometimes be achieved through application of functions like tanh or sigmoid. ReLU remains a popular choice because it can train the network faster without any major penalty to generalization accuracy. Spatial variance or flexibility is an important requirement of any image classification algorithm. This is addressed by the pooling layer. Pooling helps to reduce the number of required parameters, the computational cost and controls overfitting. The most common choice in pooling is max pooling. In max pooling, the input image is partitioned into a set of disjoint areas. The outputs of each area are the maximum value in each area. This makes a smaller size with fewer parameters and accounts for distortion. Flattening converts the pooled feature map into a sequential column of numbers (a single row or column vector). This allows that information to become the input layer of an artificial neural network for further processing. At this stage, an artificial neural network (ANN) is added to the convolutional neural network. The ANN combines the extracted features into more attributes which help predict the classes with higher accuracy. The fully connected layer is a traditional multilayer perceptron (MLP). The output layer classifier is usually a softmax [50] activation function. The softmax function takes a vector of scores and assigns values between 0 and 1 that add up to 1. Application of the softmax function can be followed by application of the loss function. Cross entropy method [52] is often applied with softmax. Minimization of the loss function leads to maximization of the performance of the network. Accuracy and loss functions after implementation of the CNN in this study are shown in Figs. 2 and 3 and elaborated in the subsequent paragraph.

Figure 4 shows the search pipeline steps. The pipeline can be viewed to have two main parts. The first part is geared toward glitch removal from the data using MuLaSE and the CNN components, while the second part deals with an actual search following the data thus processed. The analysis starts with reading the raw strain data from the LIGO Hanford (H1) and Livingston (L1). This step is followed by conditioning the data that involves bandpassing to remove the seismic wall in the lower frequencies and the shot noise in the higher frequencies and then resampling the data to $4096 \mathrm{~Hz}$. This is followed by whitening the data and removal of narrowband noise. The conditioned data then enters the MuLaSE part of the analysis where an educated estimate of signals embedded in the data are processed. The output from MuLaSE is now applied as input to the cWB pipeline which generates a number of background glitches as output. Each background glitch thus recorded is converted into a timefrequency image. In the next part of the analysis, CCSN simulated waveforms from the Kuroda 2017 and Ott 2013
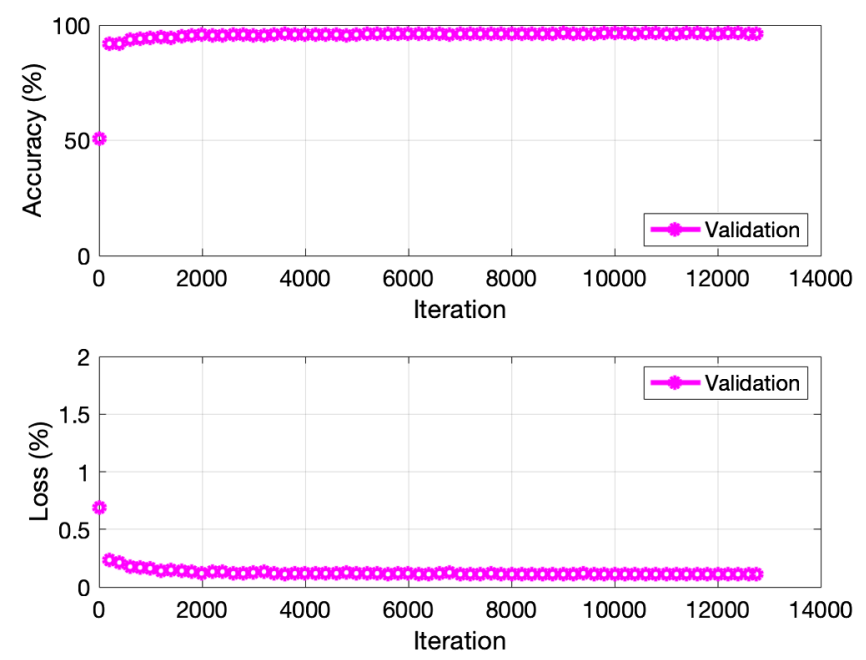

FIG. 2. The figure shows accuracy of training on the glitches and the Ott 2013 waveform.

catalogs are injected into the LIGO second observation run (O2) noise. This analysis follows a similar path to the previous one, the difference being that the output is a set of CCSN signals that had been injected. The CNN is now trained on the background glitches as well as on the recovered CCSN signals. To test the efficiency of the trained CNN, a new test data set is selected and glitches produced in from this data set are classified. It has been noted that the classification accuracy is $\geq 90 \%$. Figures 2 and 3 demonstrate the validation of the CNN. For Kuroda 2017 s11 waveforms,21478 background glitches and 21446 signals have been used to train the network. Validation has been done using 7810 glitches and 8051 signals. The accuracy rises to $90 \%$ within 1000 iterations and the loss drops to $\leq 0.5 \%$ within the same number of iterations. For Ott 2013 waveforms, 21478 background glitches and 22385 signals have been used to train the network. Validation has been done using 7810 glitches and
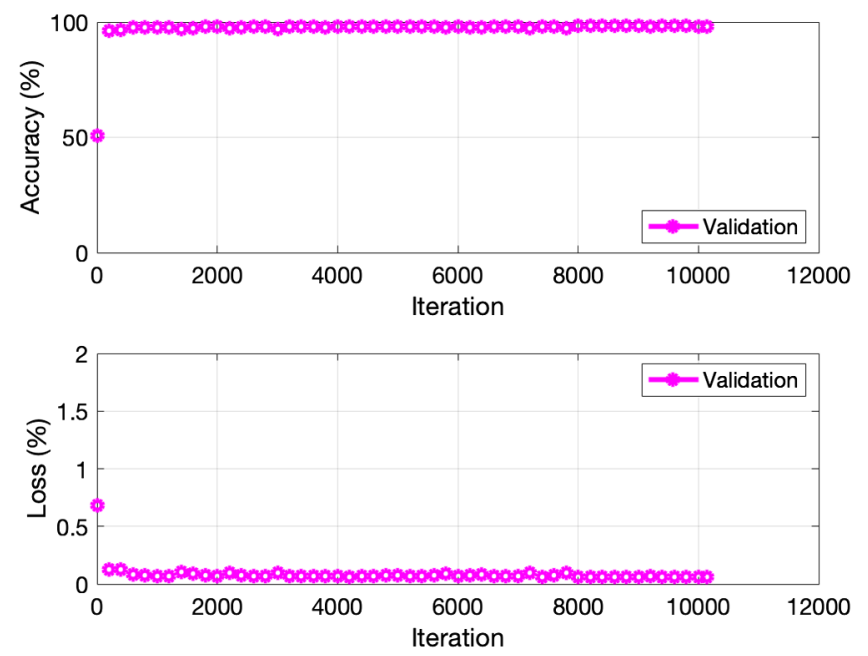

FIG. 3. The figure shows Loss of accuracy vs the number of iterations on the glitches and the Kuroda 2017 s11 waveform. 

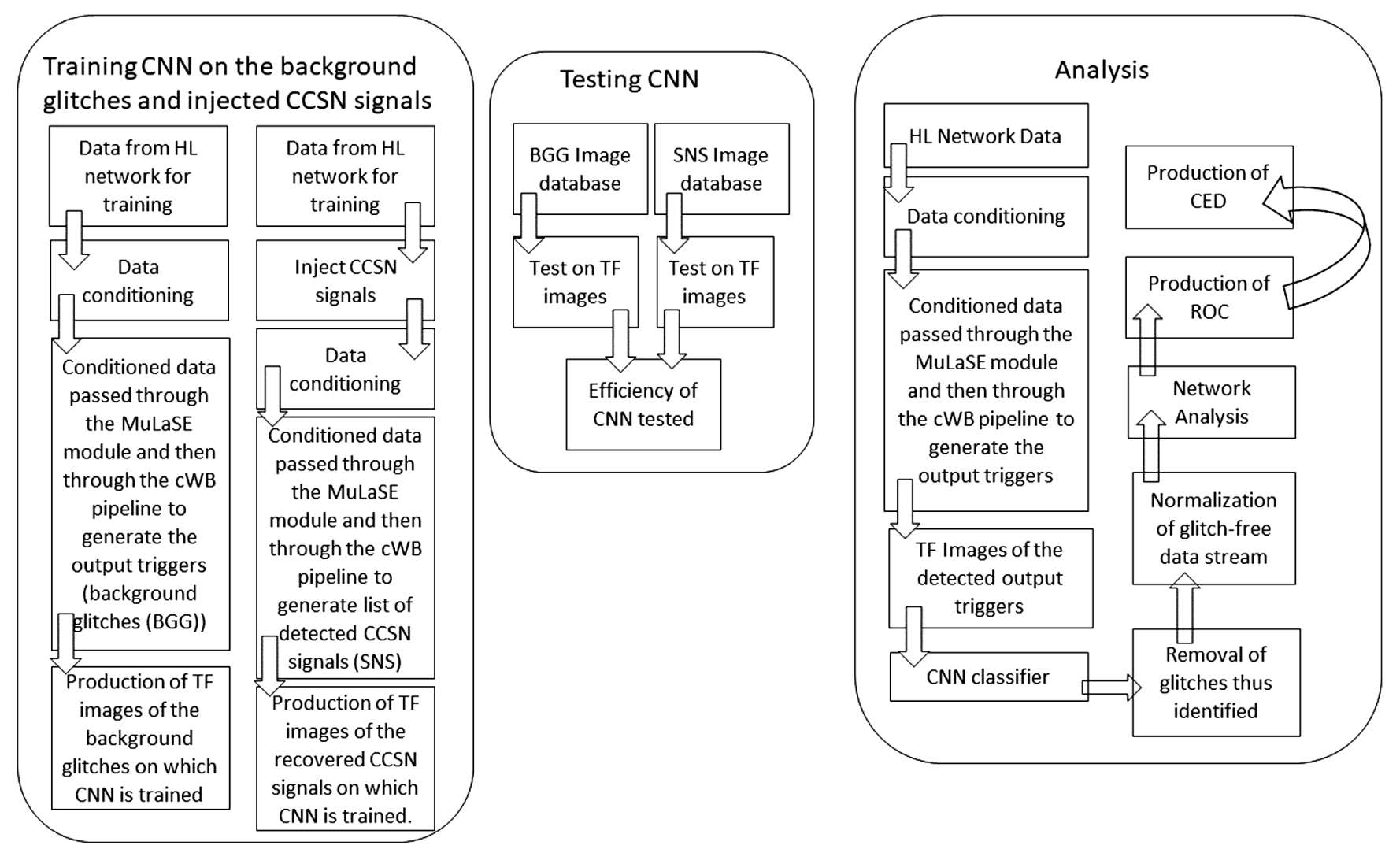

FIG. 4. The search pipeline is shown here. The pipeline can be viewed to have two main parts. The two left side boxes represent the identification of glitches, training and testing of the CNN components and classification, while the second part deals with an actual detection and parameter estimation resulting from data with glitches removed. Details of the pipeline is given in the Search Algorithm and Pipeline section.

7999 signals. As before, the accuracy rises to $90 \%$ within 1000 iterations and the loss drops to $\leq 0.5 \%$ within the same number of iterations.

At this stage, the analysis is ready for the detection of a GW signal. It starts with a data streams from the detectors with an injected signal and follow the MuLaSE $+\mathrm{cWB}$ steps of the analysis pipeline. Once the glitches have been identified, it is now time for them to be removed from the

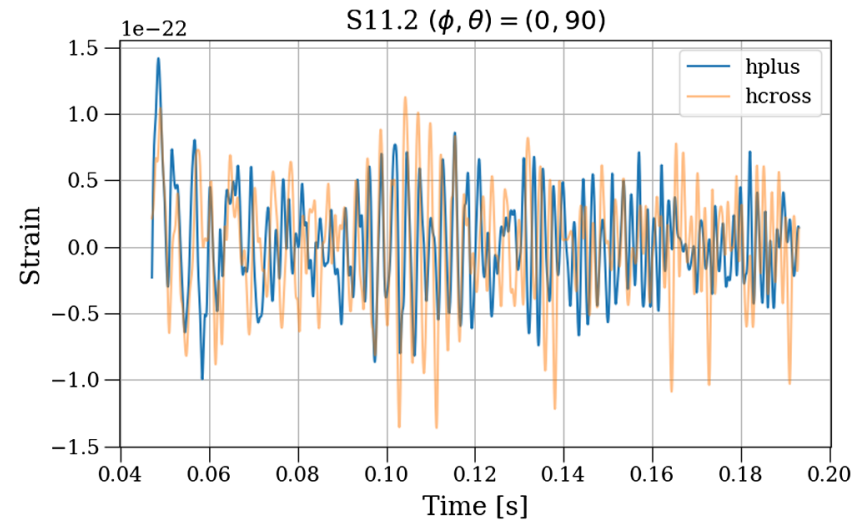

FIG. 5. The figure shows the $h_{+}$and $h_{x}$ components of the Kuroda 2017 S11 waveform. The waveform is $0.16 \mathrm{~s}$ in duration. data. In the current study, this is achieved by the method of statistical imputation [53-58] for glitch removal. In statistical data analysis, imputation is the process of replacing missing data with an estimated value based on other available information. Imputation methods run the risk of introducing a bias unless one is careful. In order to deal with the problem of increased noise due to imputation, Rubin [56] developed a method for averaging the outcomes

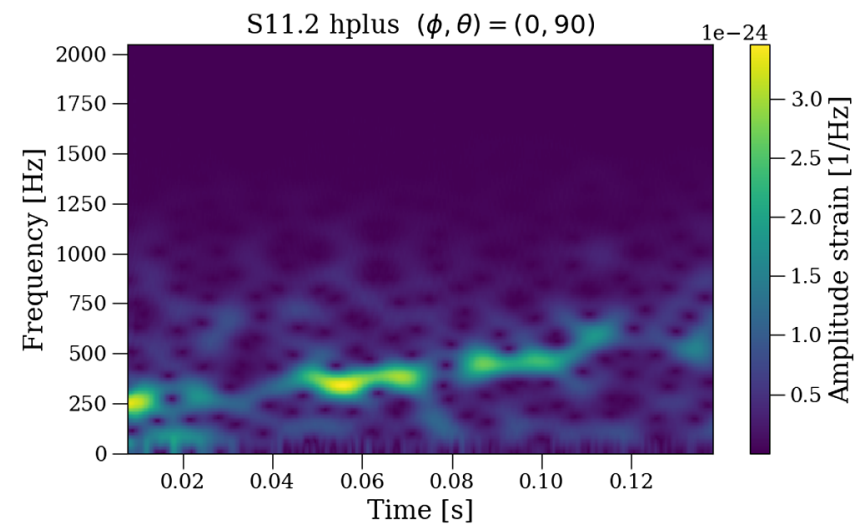

FIG. 6. The figure shows the spectrogram of the Kuroda 2017 S11 waveform. Only $h_{+}$is seen here. 


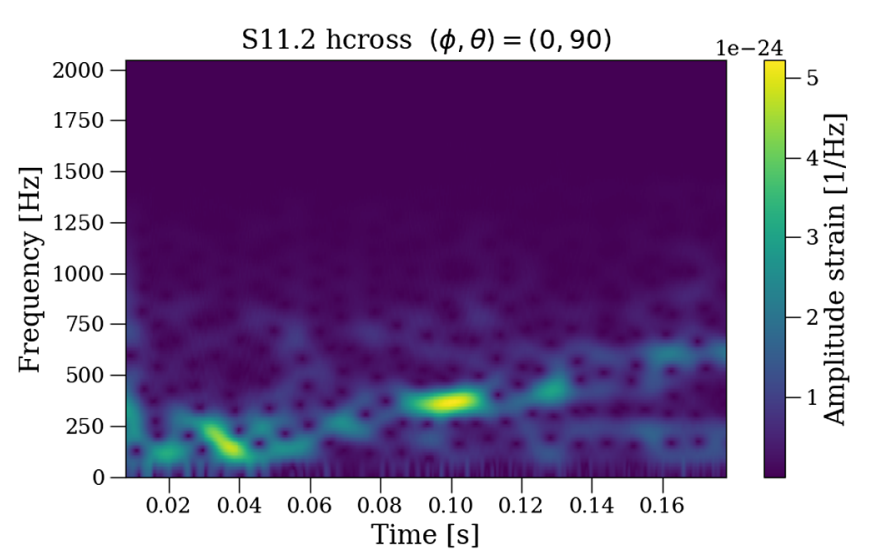

FIG. 7. The figure shows the spectrogram of the Kuroda 2017 S11 waveform. Only $h_{X}$ is seen here.

across multiple imputed data sets to account for this. This process is known as the method of multiple imputation that involves imputed values to be drawn $m$ times from a distribution. At the end of this step, there should be $m$ completed data sets. At the end of this step there should be $m$ analyses results that are consolidated into one result by calculating the mean and variance by combining simulations from each separate model. The resulting data set is a clean time series from which glitches identified by the CNN have been removed. This time series is now provided as an input to the cWB pipeline. The network analysis ends with the production of background and detected events from which the receiver operating characteristics (ROC) are generated. Coherent event displays (CED) are also generated that provide timefrequency plots of the detected events and the waveform reconstructions.

\section{RESULTS}

The results of this analysis were obtained using a 26 hour data segment from O2. Figure 5 shows the Kuroda 2017 S11 waveform at $10 \mathrm{kpc}$. The $h_{+}$and $h_{x}$ components are

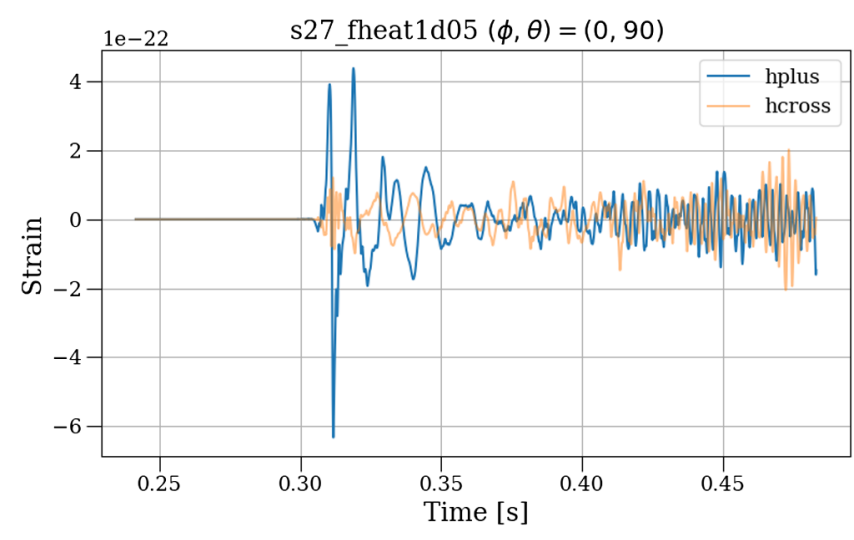

FIG. 8. The figure shows the $h_{+}$and $h_{x}$ components of the Ott 2013 S27 waveform. The waveform is $0.16 \mathrm{~s}$ in duration.

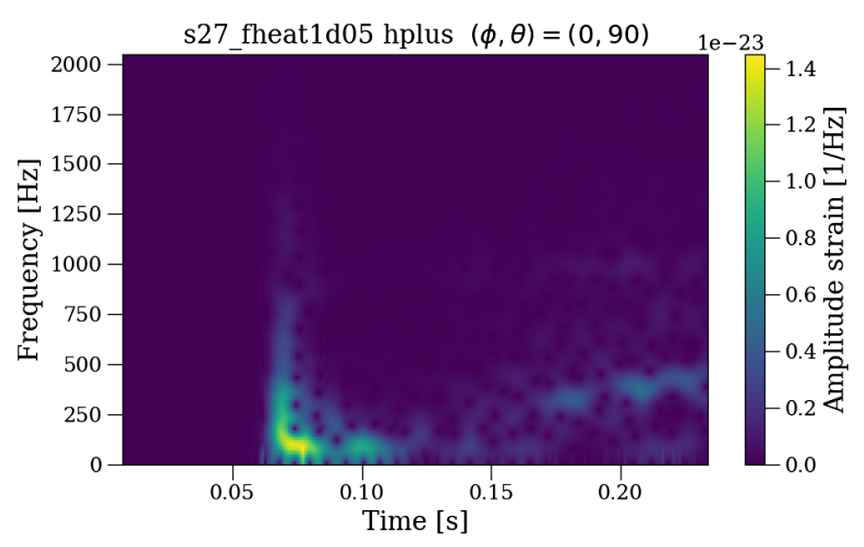

FIG. 9. The figure shows the spectrogram of the Ott 2013 S27 waveform. Only $h_{+}$is seen here.

shown for a duration of $0.16 \mathrm{~s}$. Figures 6 and 7 show the spectrogram of the $h_{+}$and $h_{x}$ components. The SASI is clearly visible around $\sim 250 \mathrm{~Hz}$. Figures $8-10$ show the same for Ott 2013 S27 waveform respectively. The waveform duration is $0.25 \mathrm{~s}$ and a strong low frequency component is visible between 100-200 Hz. Figure 11 shows nine randomly selected glitches from the background as determined by the analysis. These glitches typically had a duration of 10-16 milliseconds and varied in frequency anywhere between $150 \mathrm{~Hz}$ to $1 \mathrm{kHz}$. Figure 11 shows the spectrograms of the injected Ott 2013 s27 waveforms as picked up by the pipeline. These spectrograms display the characteristics of the waveform as shown in Figs. 12 and 10. Figure 13 shows the spectrograms of the injected Kuroda 2017 s11 waveforms as picked up by the pipeline. These spectrograms display the characteristics of the waveform as shown in Figs. 6 and 7.

Figures 14 and 15 show the receiver operating characteristics of the two waveforms under study. In case of Ott 2013 waveform, in a range of FAR below $10^{-6}$, the efficiencies of the MuLaSECC and the cWB pipelines are practically the

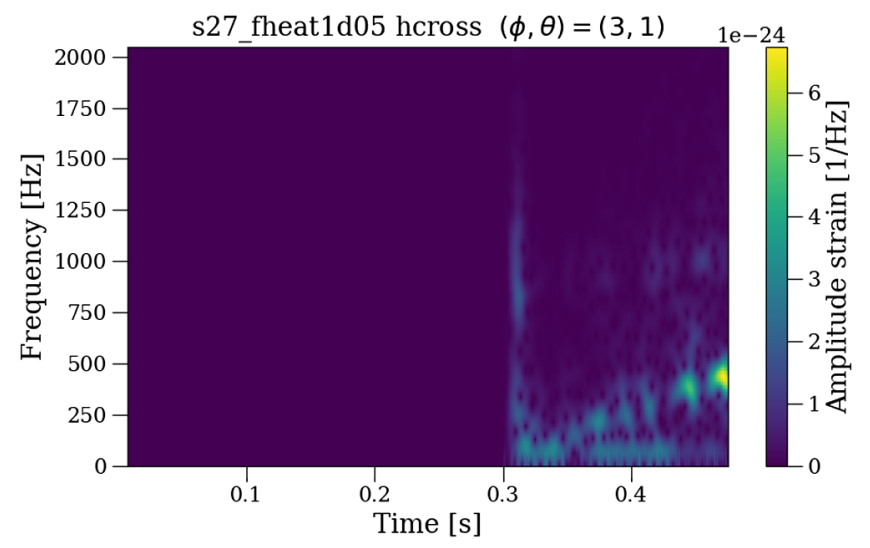

FIG. 10. The figure shows the spectrogram of the Ott 2013 S27 waveform. Only $h_{X}$ is seen here. 

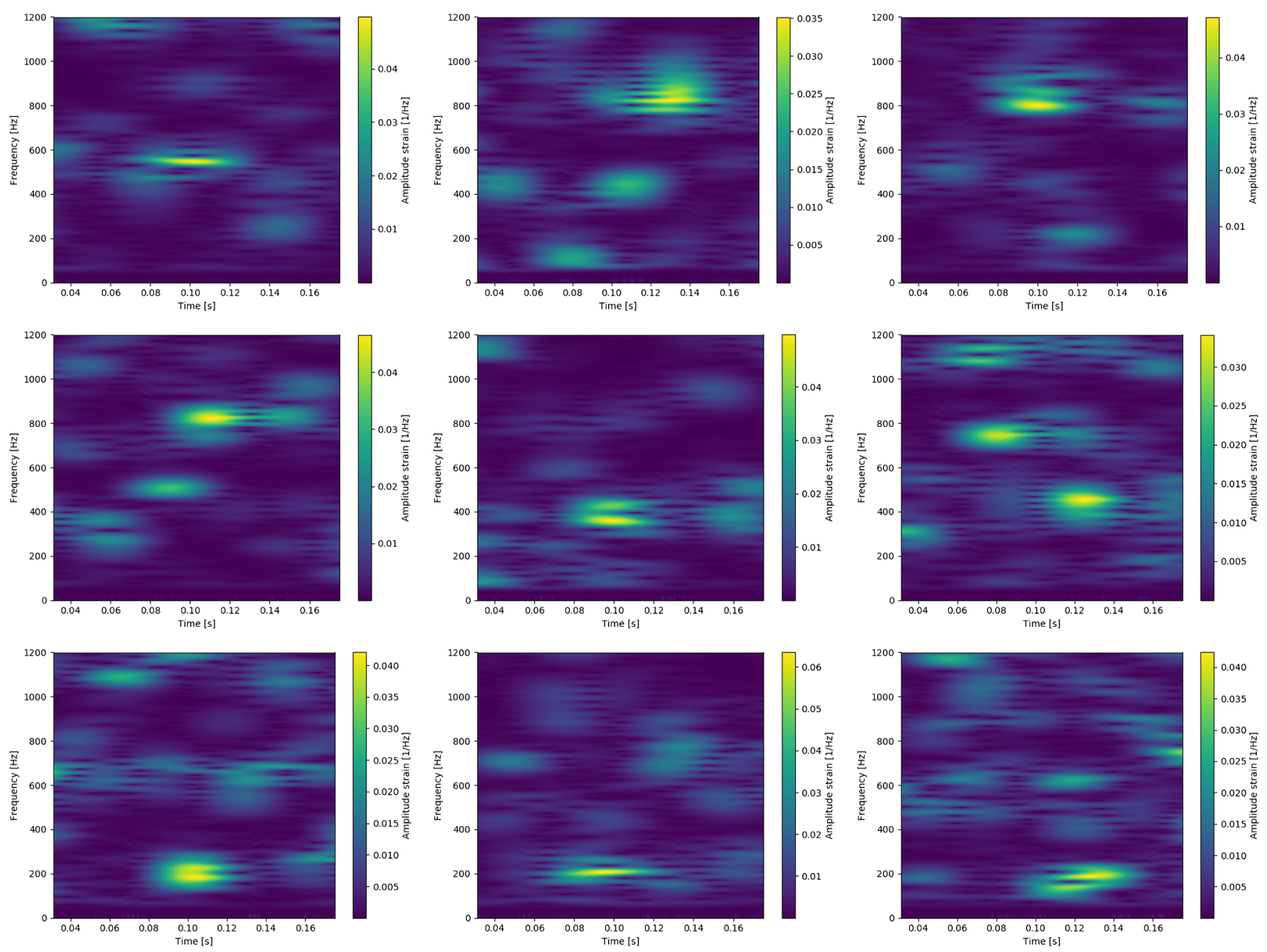

FIG. 11. The figure shows spectrograms of the different types of background glitches obtained in the analysis. These glitches were identified to be non-CCSN signals by the trained CNN.

same, with the MuLaSECC efficiency marginally better. However, above FAR $10^{-6}$, the cWB pipeline's performance efficiency is higher by about $5 \%$. In case of the Kuroda 2017 s11 waveform, the MuLaSECC pipeline operates between 5 and $8 \%$ higher efficiency than the cWB pipeline in a range of FAR between $10^{-7}$ and close to $10^{-5}$ at which point it becomes almost equal.

The last stage of the analysis involves injection of the Kuroda 2017 s11 and Ott 2013 s27 waveforms into the O2 data (20 injections/600s data with varying SNR's) and studying the events detected at the end of the cWB and the MuLaSECC pipelines. We used SNR's in the range 5 to 20 with the following steps: 5, 7, 8, 10, 15 and 20 . With the Ott 2013 waveform, the cWB pipeline did not detect an output event for SNR's 5, 7 and 8. However, the MuLaSECC pipeline detected the SNR 8 injection. Figure 16 shows the spectrogram of signal detected at an output SNR of 7.8 by the MuLaSECC pipeline for the L1 (bottom panel) and the H1 (top panel) detectors.
Figure 17 shows the reconstruction of the detected signal waveform at the $\mathrm{H} 1$ and $\mathrm{L} 1$ detectors. In this figure, the $\mathrm{L} 1$ results are in the left hand column and the $\mathrm{H} 1$ results are in the right hand column. The top panels show the bandlimited signal with the original in black and the reconstructed signal in red. The middle panels show the injected (in black) vs reconstructed (in red) whitened signal time series. The bottom panels display the amplitude spectra of the injected (black) vs reconstructed (red) whitened signal data.

Figure 18 shows the reconstruction of the detected signal spectrum at the $\mathrm{H} 1$ and $\mathrm{L} 1$ detectors. In this figure, the L1 results are in the right hand column and the H1 results are in the left hand column. The top panels show the injected signal time frequency maps, while the bottom panels show the same for the reconstructed signal. It is found that the reconstructed signal as detected by the MuLaSECC pipeline has well reconstructed the low frequency $(150-300 \mathrm{~Hz})$ component of the signal, but missed 

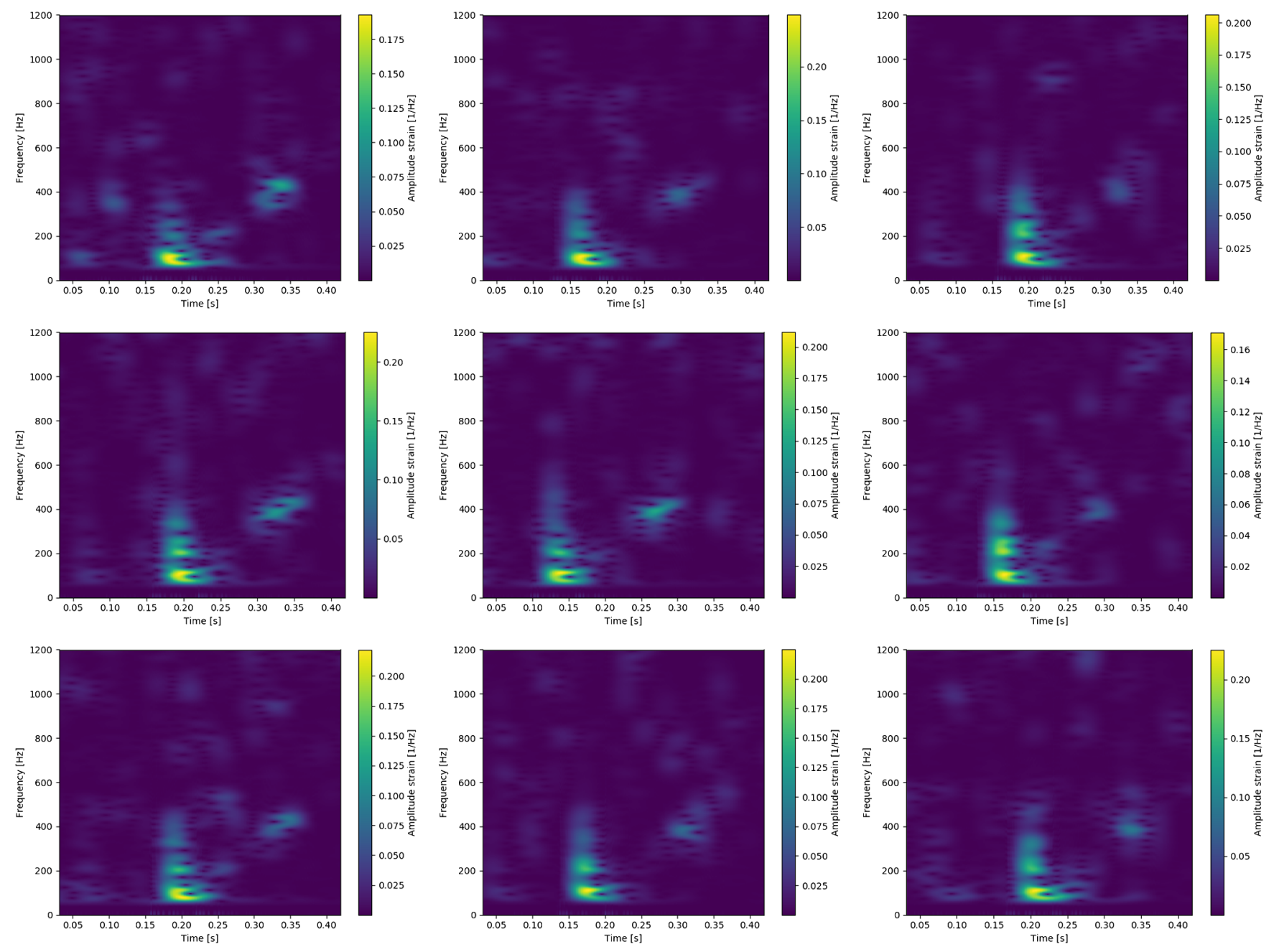

FIG. 12. The figure shows spectrograms of the Ott 2013 s27 waveforms as recovered by the pipeline.

the $400 \mathrm{~Hz}$ component. These results are still significant given that the MuLaSECC has been able to detect the signal that otherwise may have gone unnoticed. It is noted that the reconstruction is only approximately correct in amplitude and phase and represents the low frequency domain of the signal. The high frequency components are missing in the reconstructed plot. However, given the low SNR signal, the detection is significant and partial reconstruction, coupled with multimessenger observational evidence, if available, will bring together sufficient information to draw conclusions.

A somewhat similar situation is noticed with the Kuroda 2017 s11 waveforms as well. The cWB pipeline did not detect an output event for SNR's 5 and 6 . However, the MuLaSECC pipeline detected the SNR 6 injection. Figure 19 shows the spectrogram of signal detected at an output SNR of 7.8 by the MuLaSECC pipeline for the L1 (bottom panel) and the H1 (top panel) detectors. Figure 20 shows the reconstruction of the detected signal waveform at the $\mathrm{H} 1$ and $\mathrm{L} 1$ detectors.
In this figure, the L1 results are in the left hand column and the $\mathrm{H} 1$ results are in the right hand column. The top panels show the band-limited signal with the original in black and the reconstructed signal in red. The middle panels show the injected (in black) vs reconstructed (in red) whitened signal time series. The bottom panels display the amplitude spectra of the injected (black) vs reconstructed (red) Whitened Signal data. Figure 21 shows the reconstruction of the detected signal spectrum at the $\mathrm{H} 1$ and $\mathrm{L} 1$ detectors. In this figure, the $\mathrm{L} 1$ results are in the right hand column and the $\mathrm{H} 1$ results are in the left hand column. The top panels show the injected signal time frequency maps, while the bottom panels show the same for the reconstructed signal. It is found that the reconstructed signal as detected by the MuLaSECC pipeline has well reconstructed the frequencies of the signal around $200 \mathrm{~Hz}$, but missed the higher frequencies. As in case of the Ott waveforms, these results are still significant because the MuLaSECC pipeline has been able to detect the signal. 

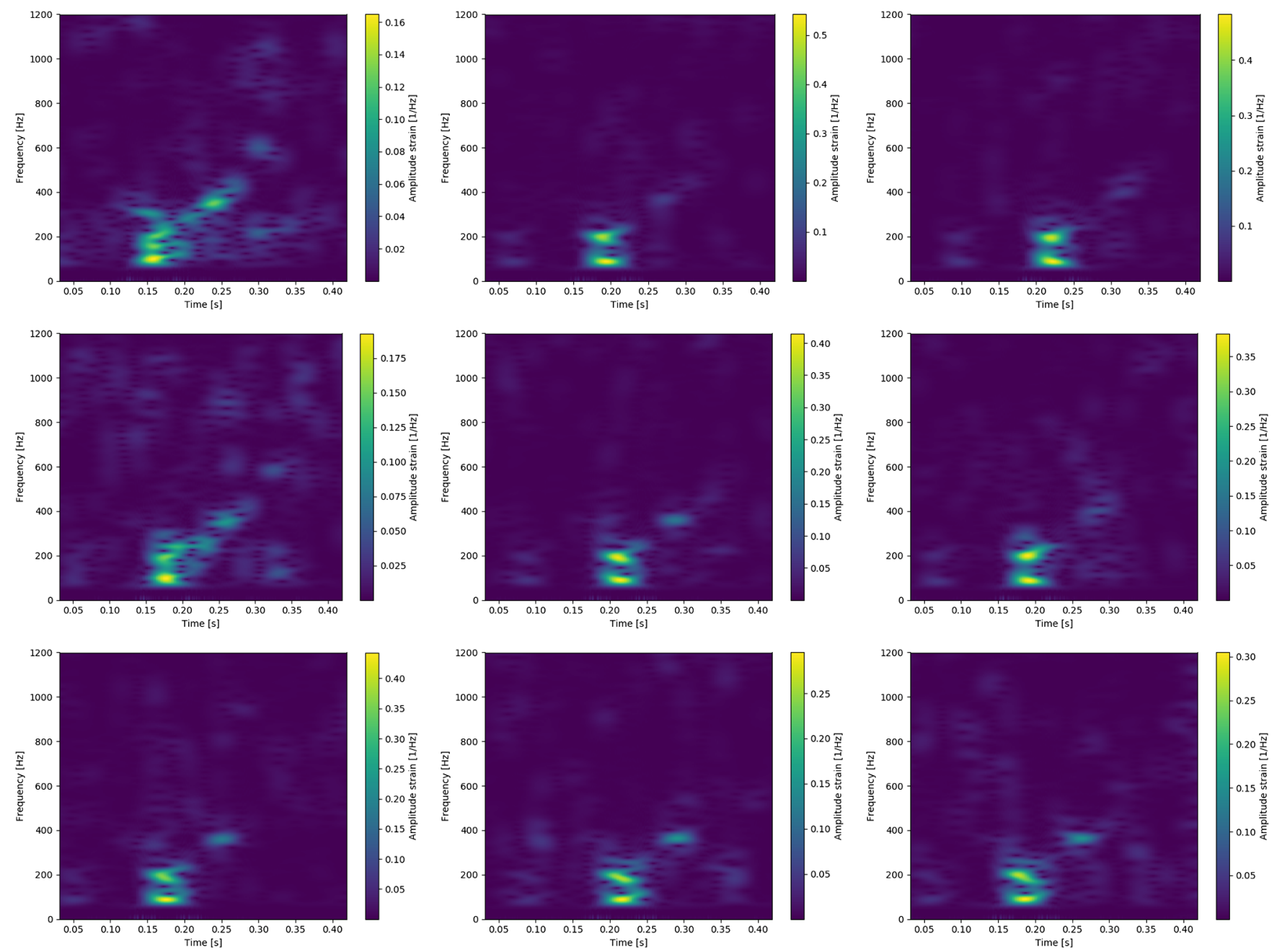

FIG. 13. The figure shows spectrograms of the Kuroda 2017 s11 waveforms as recovered by the pipeline.

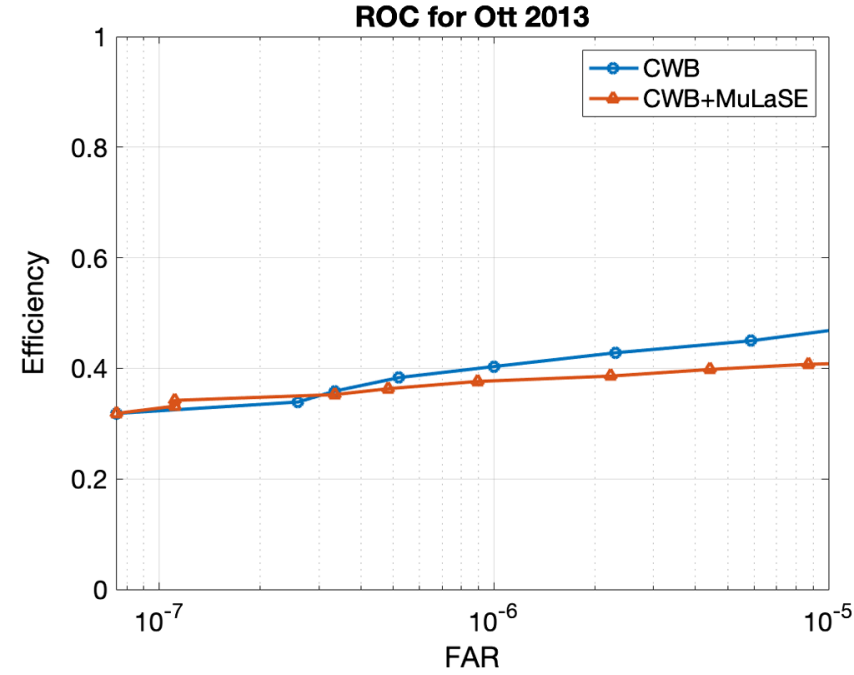

FIG. 14. The figure shows the receiver operating characteristics (ROC) of the Ott 2013 s27 waveform.

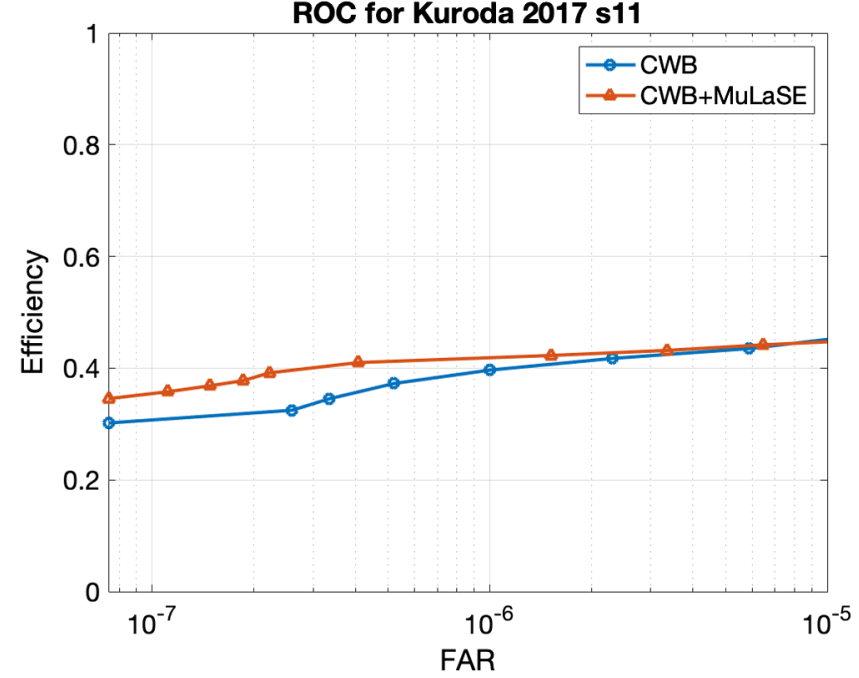

FIG. 15. The figure shows the receiver operating characteristics (ROC) of the Kuroda 2017 s11 waveform. 

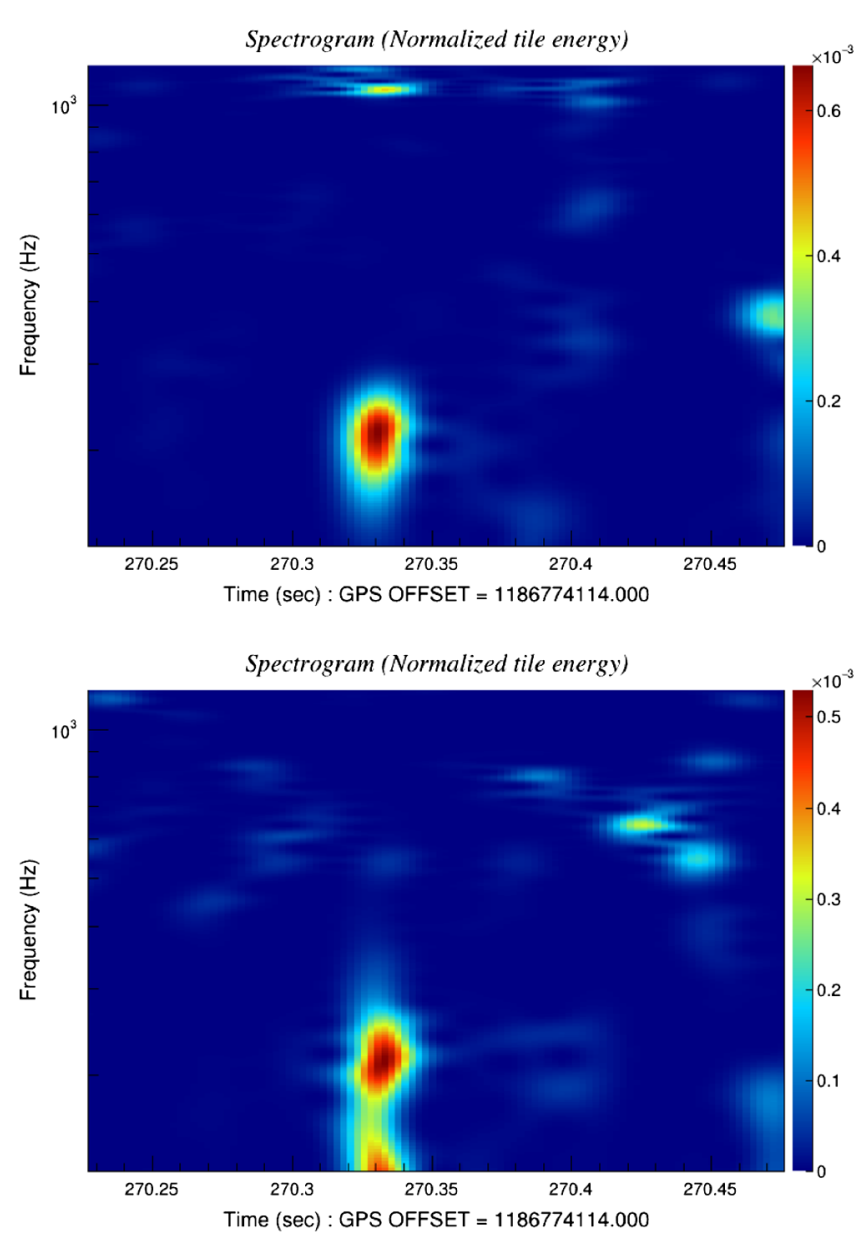

FIG. 16. This figure shows the spectrogram of the Ott 2013 s27 signal detected at an output SNR of 7.8 by the MuLaSECC pipeline for the L1 (bottom panel) and the H1 (top panel) detectors.

We want to demonstrate a comparative study using injections with SNR 10 and 15 for the two waveforms under study using the cWB and the MuLaSECC pipelines. There are three types of figures for each injected SNR. These are

(i) A set of $2 \times 2$ spectrogram plots showing the detected signal. The figures are arranged and depicted as follows. The cWB pipeline results are in the left column, with $\mathrm{H} 1$ at the top and $\mathrm{L} 1$ at the bottom. The MuLaSECC pipeline results are in the right column, with $\mathrm{H} 1$ and L1 in the same order; see Figs. 22, 25, 28 and 31.

(ii) A set of $3 \times 4$ plots with the two left hand columns showing results from the cWB pipeline and the two right hand columns showing results from the MuLaSECC pipeline. For each pipeline, the left hand column presents results from $\mathrm{H} 1$ and the right hand column present results from L1. The top panels show the band-limited signal with the original in black and the reconstructed signal in red. The middle panels show the injected (in black) vs reconstructed (in red) whitened signal time series. The bottom panels display the amplitude spectra of the injected (black) vs reconstructed (red) whitened signal data; see Figs. 23, 26, 29 and 32 .

(iii) A set of $2 \times 4$ spectrogram plots of the cWB results in the two left hand columns and the MuLaSECC results in the two right hand columns. For each pipeline, the left hand column presents results from $\mathrm{H} 1$ and the right hand column present results from L1. The top panels show the time-frequency plots for the bandlimited signal. The bottom panels display the same for the reconstructed signal; see Figs. 24, 27, 30 and 33.

Figures 22-24 show the results for a Kuroda 2017 s11 injection with SNR 10. This is detected at an output SNR of 10.6 by the cWB pipeline and at SNR 11.9 by the MuLaSECC pipeline. The MuLaSECC pipeline has been able to better reconstruct the low frequency part of the signal below $100 \mathrm{~Hz}$. A more extensive reconstruction of the duration of the signal has been picked up by the MuLaSECC pipeline. Figures 25-27 show the results for a Ott 2013 s27 injection with SNR 10. This is detected at an output SNR of 8.0 by the cWB pipeline and at SNR 17.6 by the MuLaSECC pipeline. It has been able to reconstruct the low frequency part of the signal below $100 \mathrm{~Hz}$. This has been missing in the cWB reconstruction. A more extensive reconstruction of the duration of the signal as picked up by the MuLaSECC pipeline has been demonstrated. Moreover, the reconstructed spectrogram shows frequency components around $400 \mathrm{~Hz}$ that has not been reconstructed in the cWB analysis.

Figures 28-30 show the results for a Kuroda 2017 s11 injection with SNR 15. This is detected at an output SNR of 14.7 by the cWB pipeline and at SNR 20.8 by the MuLaSECC pipeline. It is important to note that the MuLaSECC pipeline has been able to reconstruct the low frequency part of the signal below $100 \mathrm{~Hz}$. In addition, the amplitude spectrum seems to have a stronger feature between the $500-600 \mathrm{~Hz}$ range. This has been missing in the cWB reconstruction. These plots also demonstrate a more extensive reconstruction of the duration of the signal as picked up by the MuLaSECC pipeline. Figures 31-33 show the results for an Ott 2013 s27 injection with SNR 15. This is detected at an output SNR of 12.1 by the cWB pipeline and at SNR 15.0 by the MuLaSECC pipeline. It has been able to reconstruct the low frequency part of the signal below $100 \mathrm{~Hz}$. This has been missing in the cWB reconstruction. A more extensive reconstruction of the duration of the signal as picked up by the MuLaSECC pipeline has been demonstrated. 

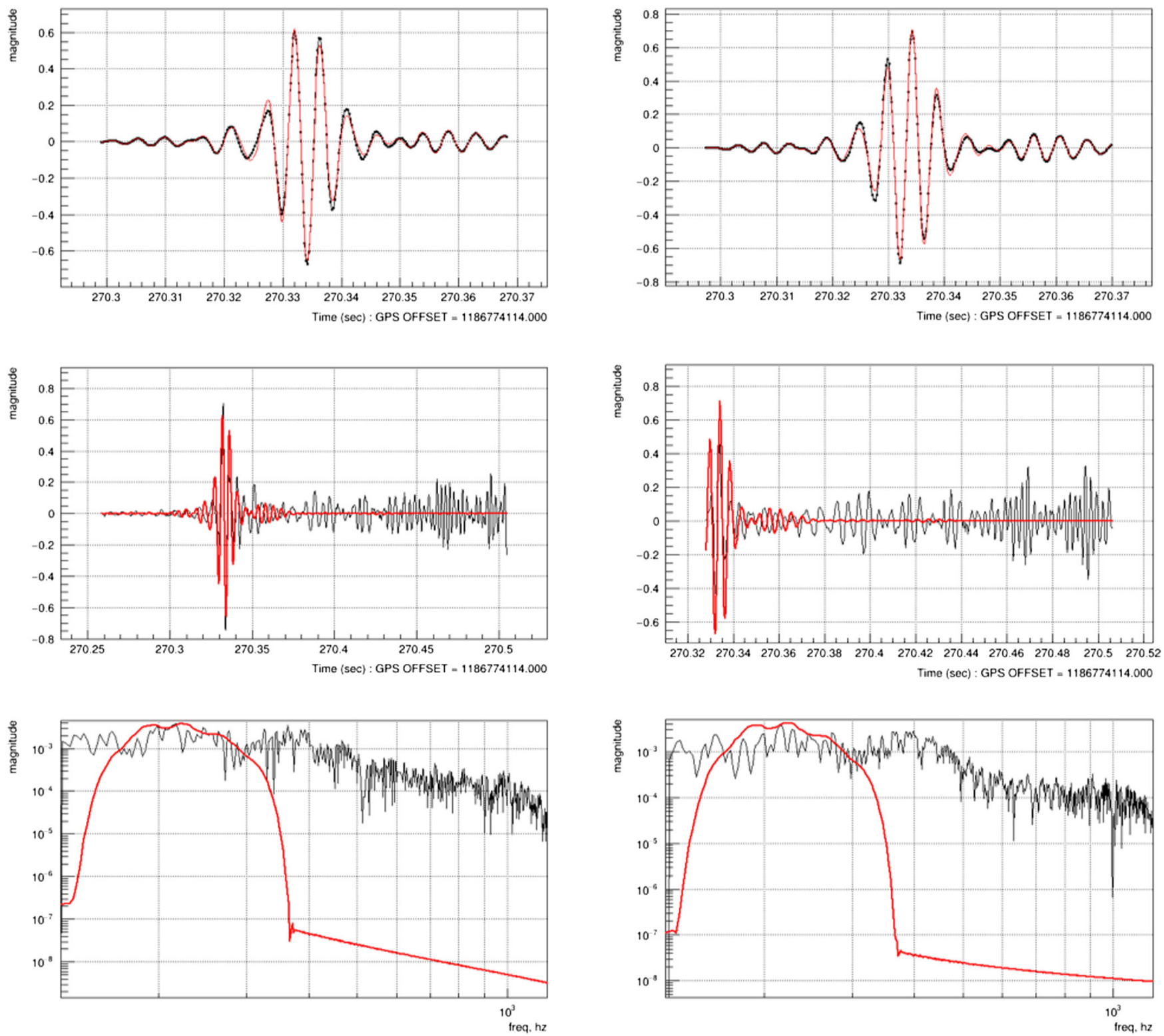

FIG. 17. The figure shows the reconstruction of the detected Ott 2013 s 27 signal waveform at the H1 and L1 detectors. In this figure, the L1 results are in the left hand column and the H1 results are in the right hand column. The top panels show the band-limited signal with the original in black and the reconstructed signal in red. The middle panels show the injected (in black) vs reconstructed (in red) whitened signal time series. The bottom panels display the amplitude spectra of the injected (black) vs reconstructed (red) whitened signal data.

\section{DISCUSSION AND CONCLUSION}

The study focused on development and implementation of a search pipeline that will increase the probability and efficiency of detecting GW signals from CCSN. The pipeline is based on a multilayer signal estimation algorithm that works in an integrative manner with the cWB pipeline to achieve a higher detection SNR at the output, with corresponding reduction in false alarm using the $\mathrm{CNN}$ that is amalgamated with the search pipeline. The results obtained are of high significance for several reasons. These include detection of weak signals (in the SNR range of 6-7) by the MuLaSECC pipeline. These input signals eluded the cWB. In addition, the signals detected at the output of the MuLaSECC pipeline have consistently higher SNR for all ranges of injected signal strengths. A notable result is the ability of the MuLaSECC pipeline to reconstruct the signals over a much broader range of frequencies, especially in the low frequency region that is of utmost importance in case of evolution of the GW signals from CCSN. Looking at the reconstructed signals in a time-frequency plane, it is found that the 

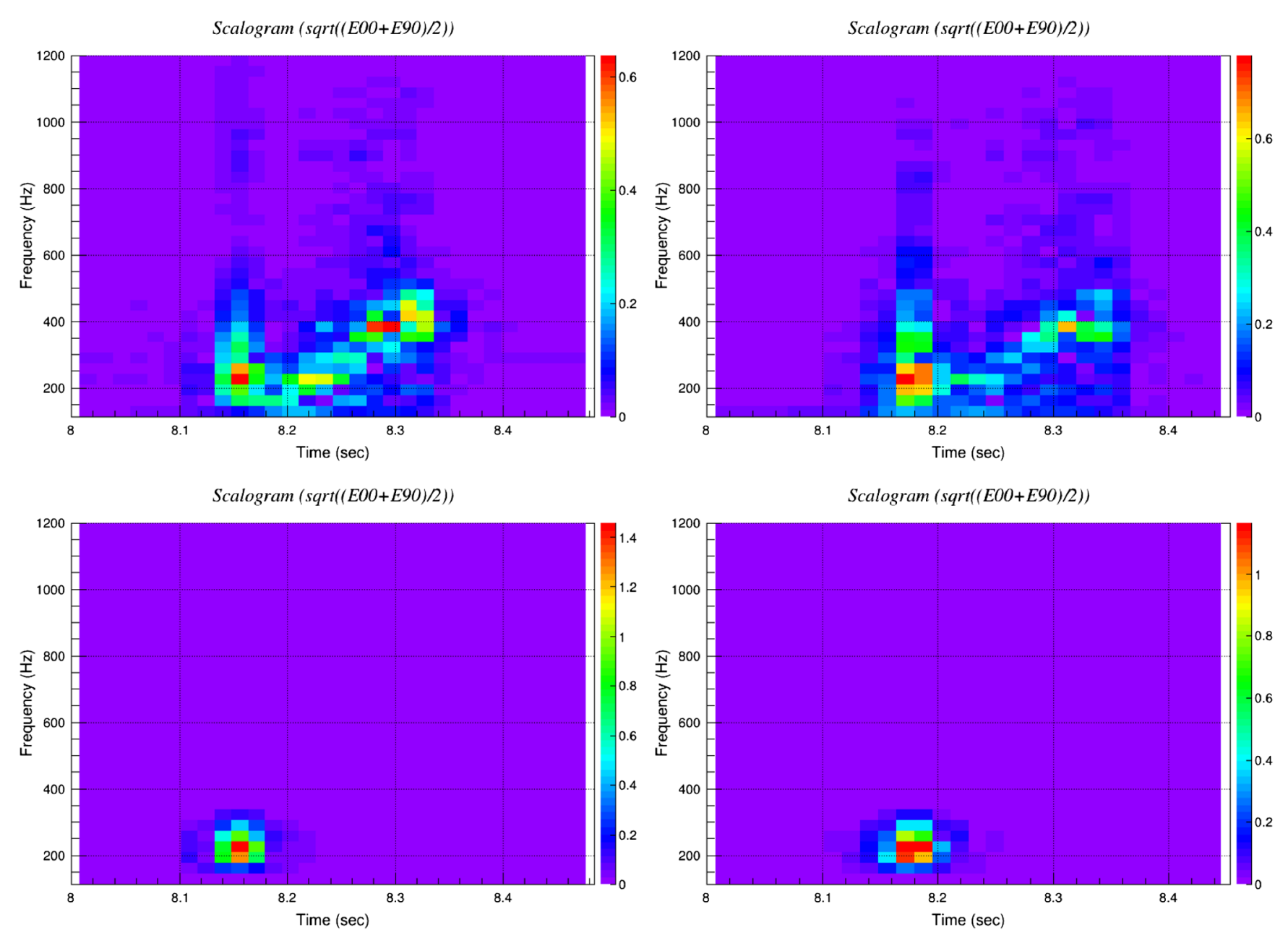

FIG. 18. The figure shows the reconstruction of the Ott 2013 s27 detected signal spectrum at the H1 and L1 detectors. In this figure, the L1 results are in the right hand column and the H1 results are in the left hand column. The top panels show the injected signal time frequency maps, while the bottom panels show the same for the reconstructed signal.

signals detected by the MuLaSECC pipeline are reconstructed over a longer (20-30 ms) duration that before.

The Kuroda 2017 s11 model has presented 3D-GR corecollapse simulations with approximate neutrino transport for a nonrotating progenitor of 11.2 solar masses. The SASI produced characteristic time modulations both in the neutrino and GW signals. The typical frequency was in the range of around 100-200 Hz. It has been emphasized in [23] that a GW signal reconstruction study is a most urgent task that needs to be performed to determine if the SASI-induced modulation in the GW and neutrino signals could be detected. The third generation of GW detectors can possibly observe the SASI-modulated GW signals; the neutrino signals can be detected by working neutrino detectors e.g., IceCube [59] and Super-K [60]. The neutrino burst can be used to determine the core bounce time, and this multi-messenger information can increase the detection confidence of any CCSN GW candidates. Thus, the method discussed in the current paper with its higher detection probability and significantly improved signal reconstruction help infer the CCSN internal dynamics (e.g., the SASI) by observing the following features:

(i) the low frequency around $100 \mathrm{~Hz}$ modulation in both GW and neutrino signal and

(ii) a $10 \mathrm{~ms}$ time delay of the SASI-modulated GW signal from the SASI-modulated neutrino event rate. Even a nondetection of the correlation could be hypothetically used to constrain the nuclear EOS's. From the limited number of the EOS used in generating these waveforms, one cannot obtain any quantitative conclusion. The demonstrated improved performance of MuLaSECC is crucial to address this urgent scientific observation during the upcoming $\mathrm{GW}$ detector observation runs.

The Ott 2013 s27 model [24] represents a 3D generalrelativistic core collapse and post-bounce simulations of the 

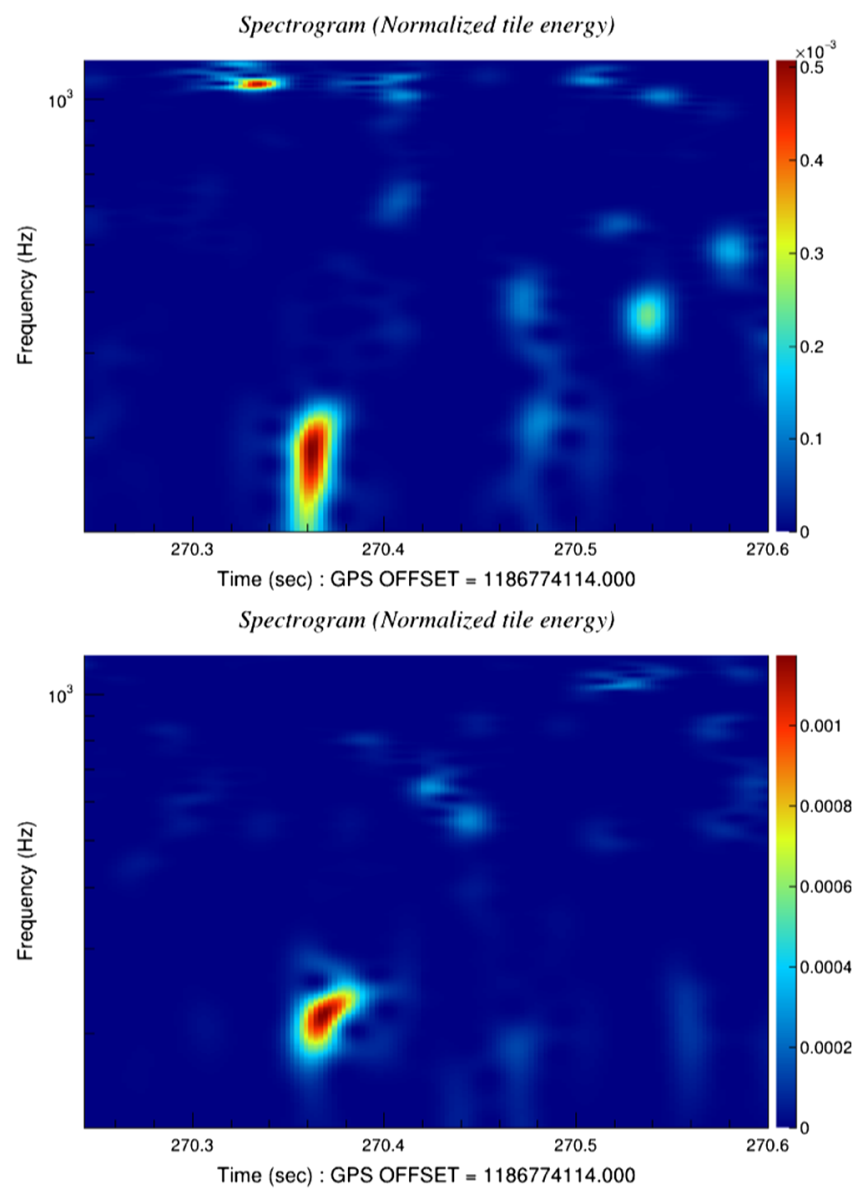

FIG. 19. This figure shows the spectrogram of the Kuroda 2017 s11 signal detected at an output SNR of 7.8 by the MuLaSECC pipeline for the L1 (bottom panel) and the H1 (top panel) detectors.

27 solar mass progenitor including the effect of variations in neutrino heating on the 3D post-bounce evolution in general and on SASI in particular. These simulations neither employed an artificial inner boundary nor did they make any symmetry assumptions or approximations for the gravitational field. For neutrinos, an energy-averaged three species neutrino leakage/heating scheme was used in the post-bounce phase, whose only free parameter had been a scaling factor in the energy deposition rate. Some of the results specific to this simulation include an early and strong growth of convective instability that is initially prompt, driven by the negative entropy gradient left behind by the stalling shock. Subsequently, convection is driven by neutrino energy deposition in the gain layer. Neutrino-driven convection first manifests itself in smallscale local hot and cold blobs of post-shock material. The small scale blobs combine over time to a few large, large volume high-entropy regions whose expansion pushes out the shock. While neutrino-driven convection is the fastest growing dominant instability, the Ott 2013 s27 model shows growth of periodic low-deformations of the shock front that are characteristic of the linear phase of the SASI. Furthermore, the SASI is shown to be the strongest in the model with the least neutrino heating and the weakest in neutrino-driven convection. As already stated in [23], the simultaneous observation of neutrino and GW signals will help scientists unravel the inner mechanisms of a CCSN through insight into the thermodynamics and multi-dimensional dynamics of the PNS and the postshock region. The Ott 2013 s27 model also suggests that a study the GW emission from accelerated quadrupole mass motions could be done. The frequency of GW signatures include a strong burst of GW's associated with postbounce prompt convection between 100 and $200 \mathrm{~Hz}$, followed by a higher-frequency between $400 \mathrm{~Hz}$ and a $\mathrm{kHz}$ emission, whose amplitudes are dominated by the deceleration of convective plumes at the edge of the PNS. If either prompt or neutrino-driven convection does not develop early, the GW signal would not have a strong initial burst, but rather a slow rise to smaller amplitudes at later times, when the SASI becomes strong. This is a key difference and may allow GW data analysts to distinguish between convection-dominated and SASI dominated post-bounce evolution in the next CCSN in the Milky Way.

Both waveform simulations above strongly hint at the low frequency behavior of the detected signal as a crucial discerning factor in determination of the central model driving the CCSN explosion. What is most noteworthy in this analysis is the ability of MuLaSECC to reconstruct the signal with significantly greater completeness toward the low frequency end. Thus, search for GW using the MuLaSECC pipeline is of fundamental importance. Effort will continue to be invested in investigating methods to further improve the reconstructions, especially in the higher frequency end. There is a rich repertoire of waveforms. We will analyze each of these waveforms in phases using the MuLaSECC pipeline. We intend to analyze the Kuroda 2017 [23] waveform resulting from the 15 solar mass progenitor (s15) where it has been shown by the author that the SASI effects are more pronounced. We will also be analyzing the Mezzacappa 2020 [27] waveforms, which indicate that the GW spectrum in the range $20-1000 \mathrm{~Hz}$ will be accessible for a galactic event. Given the capability of the MuLaSECC method to successfully reconstruct the detected waveforms to the very low end of the frequency spectrum, it will be an important study. Another future improvement from a technical point of view will be in the area of fast glitch removal. In the current study, the method of imputation has been used, but in future studies we will explore more sophisticated statistically proven methods like adaptive spline fitting with particle swarm optimization [61] which are computationally cheap and thus can help run the MuLaSECC analysis in minimal latency mode. 

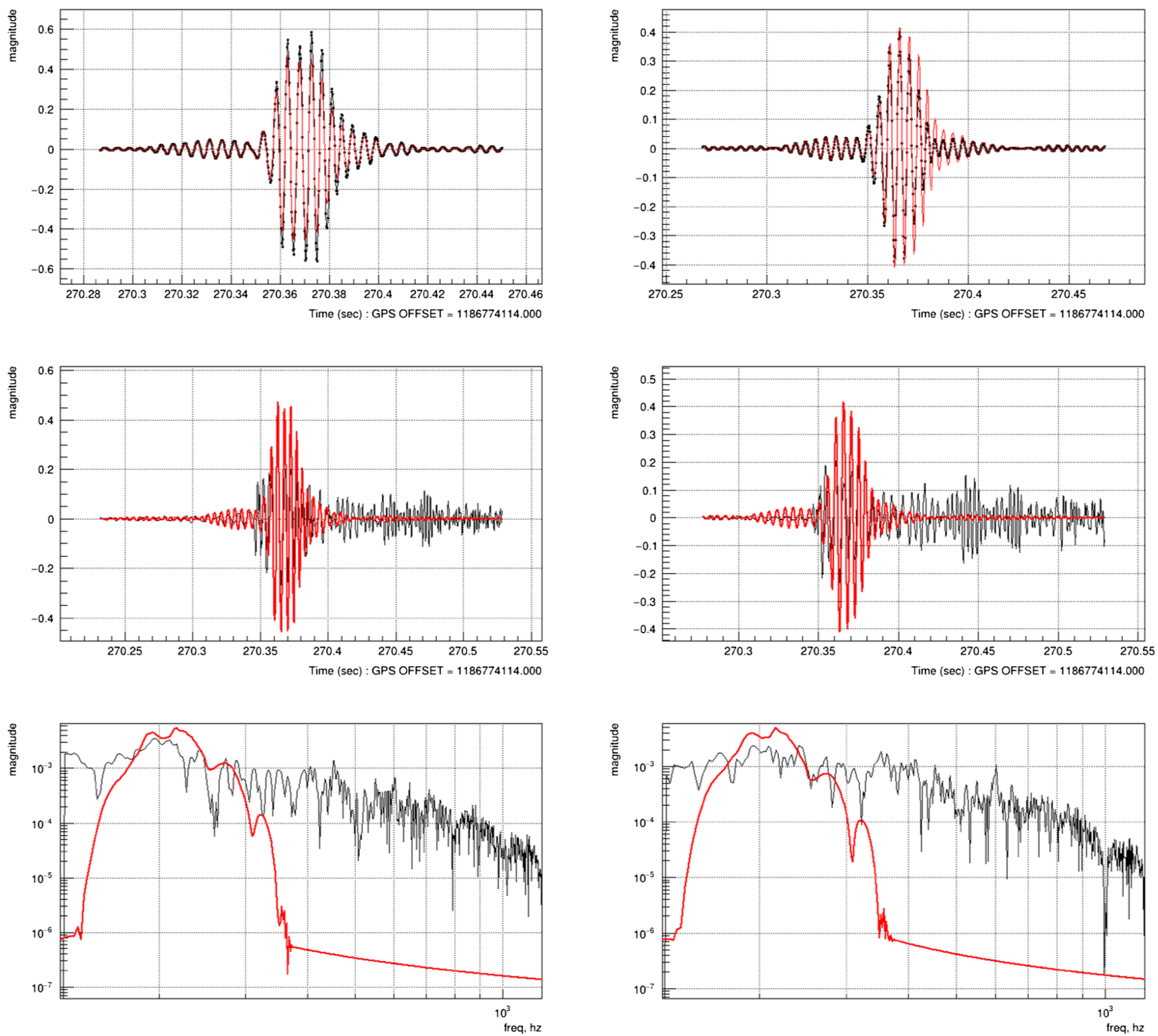

FIG. 20. The figure shows the reconstruction of the Kuroda 2017 s11 detected signal waveform at the H1 and L1 detectors. In this figure, the L1 results are in the left hand column and the H1 results are in the right hand column. The top panels show the band-limited signal with the original in black and the reconstructed signal in red. The middle panels show the injected (in black) vs reconstructed (in red) whitened signal time series. The bottom panels display the amplitude spectra of the injected (black) vs reconstructed (red) whitened signal data. 

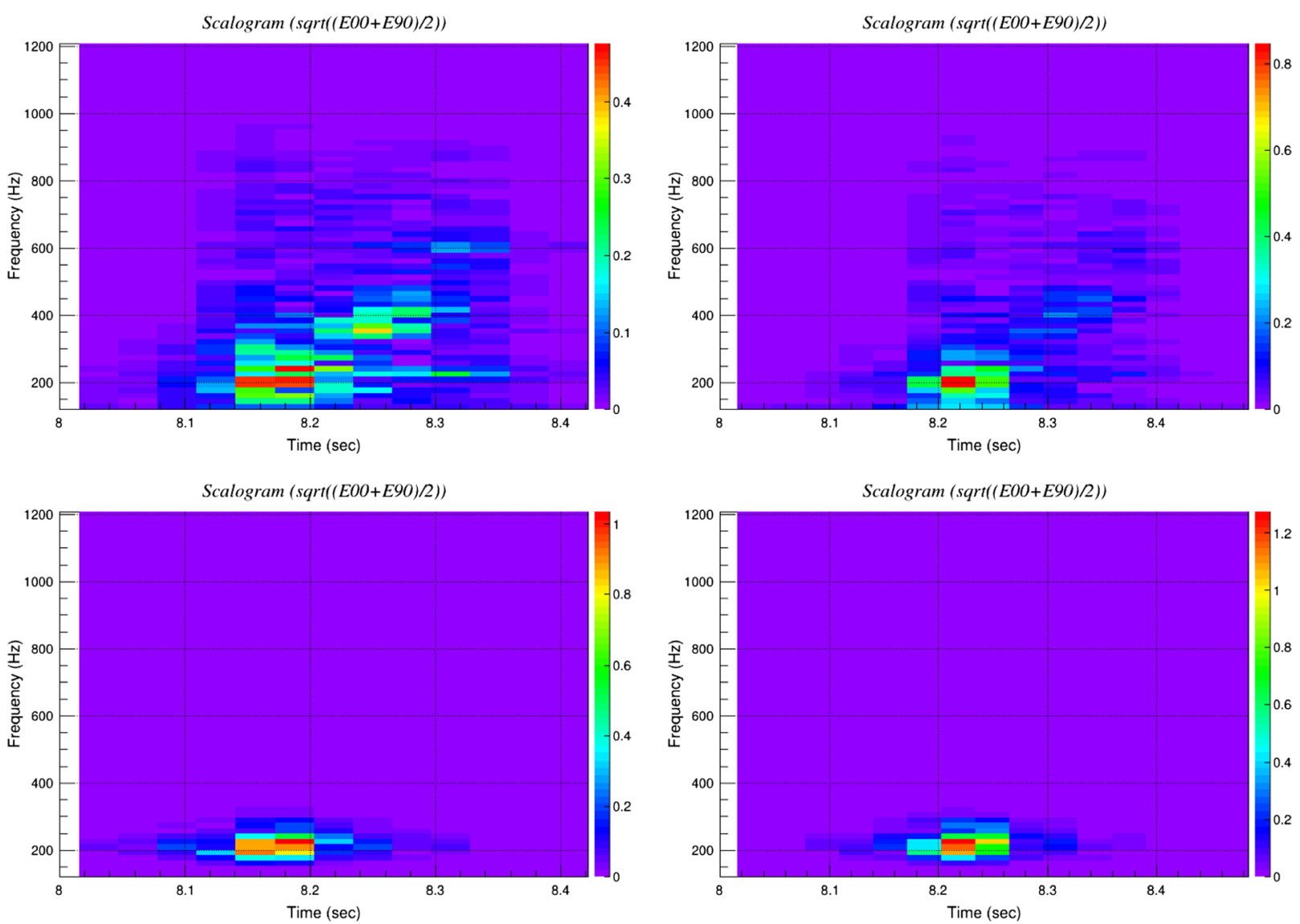

FIG. 21. The figure shows the reconstruction of the Kuroda 2017 s11 detected signal spectrum at the H1 and L1 detectors. In this figure, the L1 results are in the right hand column and the $\mathrm{H} 1$ results are in the left hand column. The top panels show the injected signal time frequency maps, while the bottom panels show the same for the reconstructed signal.
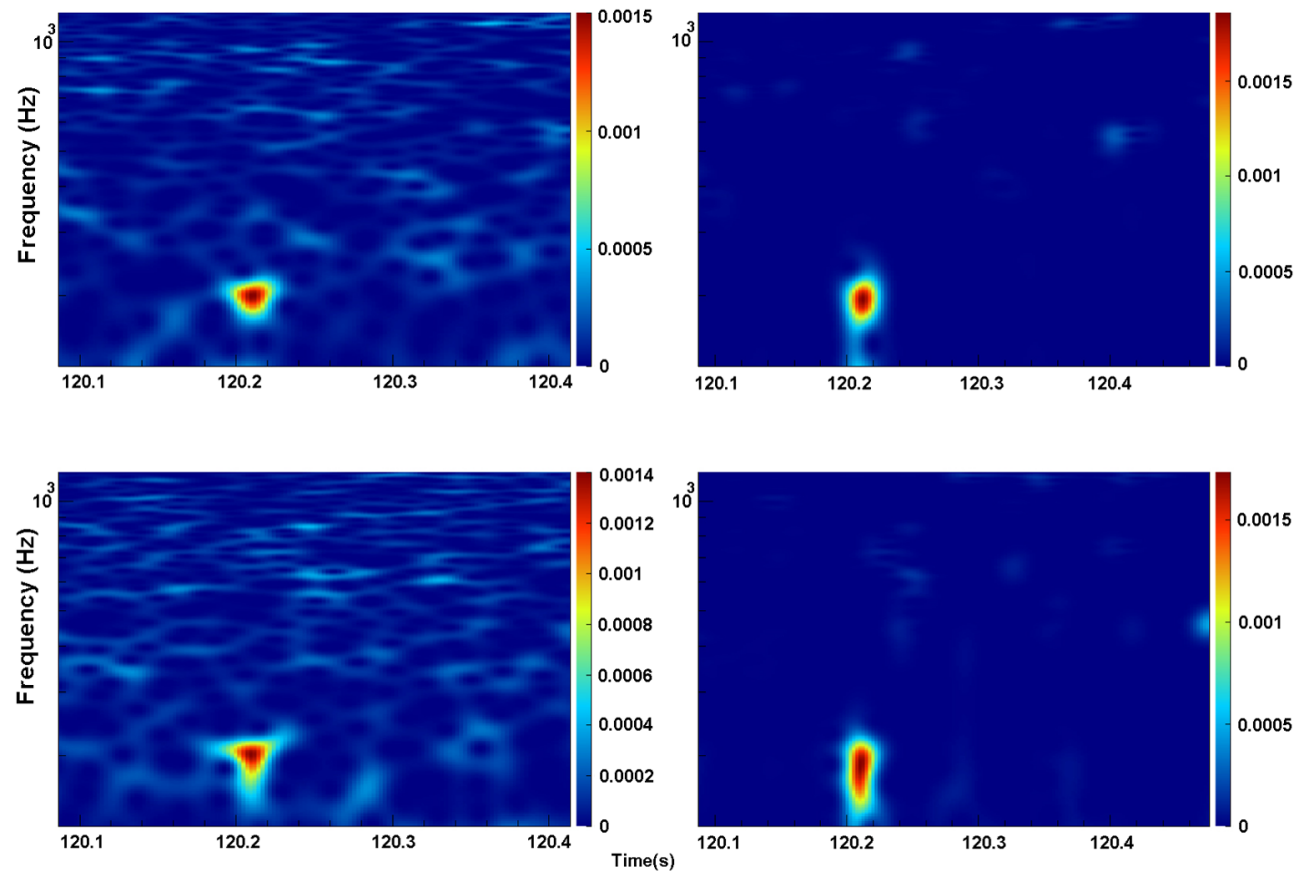

FIG. 22. This figure shows the spectrogram of signal detected at an output SNR of 10.6 by the cWB pipeline (left column, H1 at the top and L1 at the bottom); MuLaSECC pipeline (right column, H1 at the top and L1 at the bottom) detected an output SNR of 11.9. The injected Kuroda 2017 s11 signal was at SNR $=10$. 

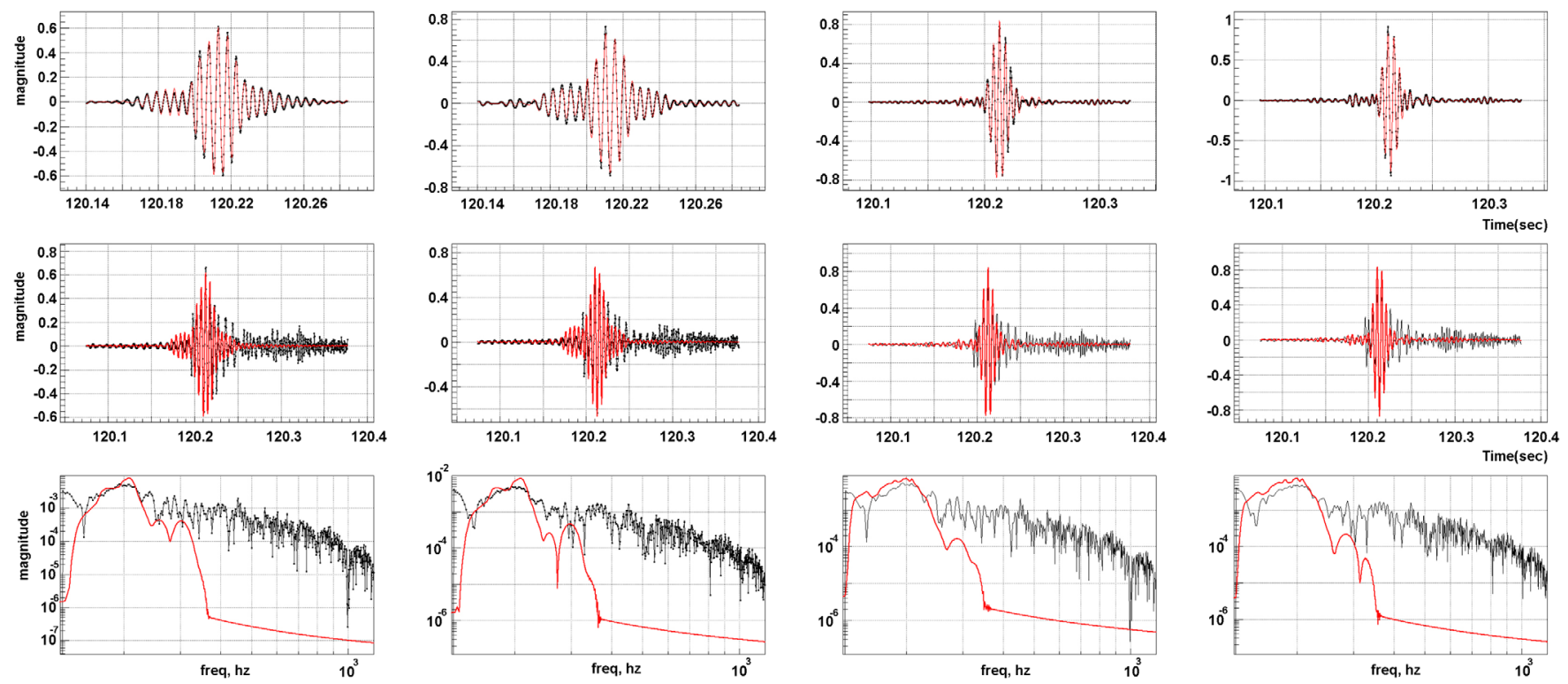

FIG. 23. The figure shows a comparative picture of the reconstruction of the detected signal waveform at the H1 and L1 detectors for Kuroda 2017 s11 waveforms as obtained by the cWB and the MuLaSECC pipelines for an injection with SNR 10. In this figure, the cWB results are in the two left hand columns and the MuLaSECC results are in the two right hand columns. For each pipeline, the left hand column presents results from $\mathrm{H} 1$ and the right hand column present results from L1. The top panels show the band-limited signal with the original in black and the reconstructed signal in red. The middle panels show the injected (in black) vs reconstructed (in red) whitened signal time series. The bottom panels display the amplitude spectra of the injected (black) vs reconstructed (red) whitened signal data.
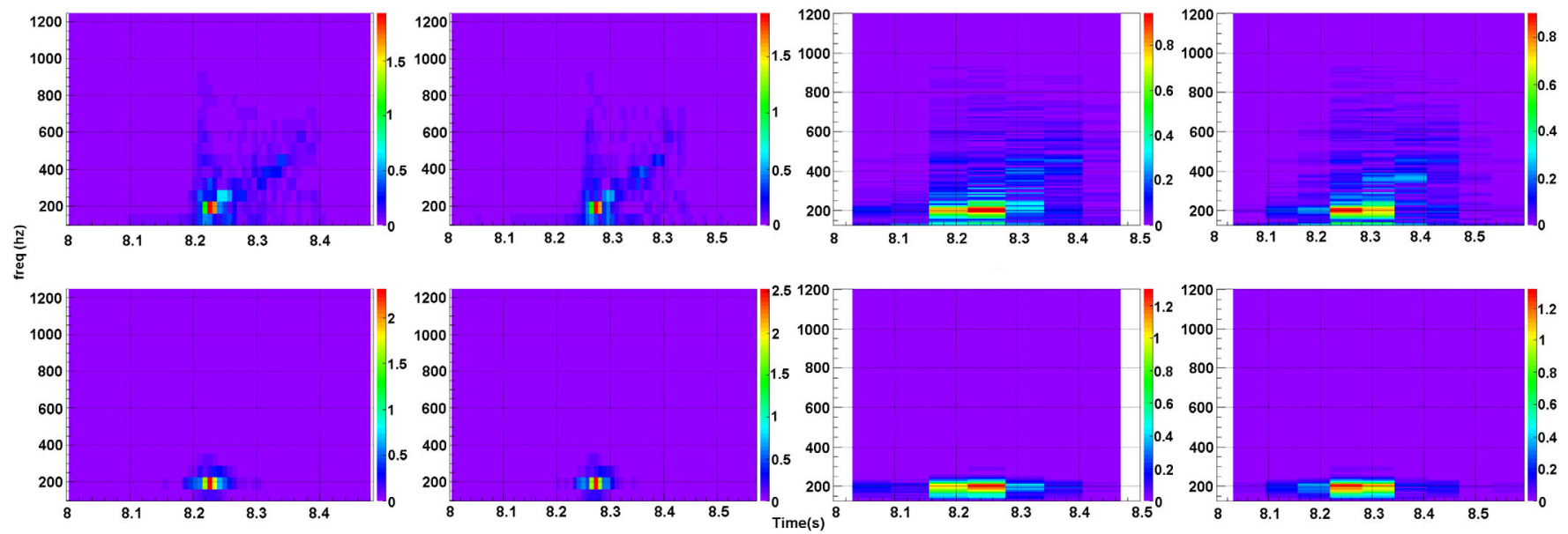

FIG. 24. The figure shows the reconstruction of the detected signal spectrum at the H1 and L1 detectors for Kuroda 2017 s11 waveforms as obtained by the cWB and the MuLaSECC pipelines for an injection with SNR 10. In this figure, the cWB results are in the two left hand columns and the MuLaSECC results are in the two right hand columns. For each pipeline, the left hand column presents results from $\mathrm{H} 1$ and the right hand column present results from L1. The top panels show the time-frequency plots for the band-limited signal. The bottom panels display the same for the reconstructed signal. 

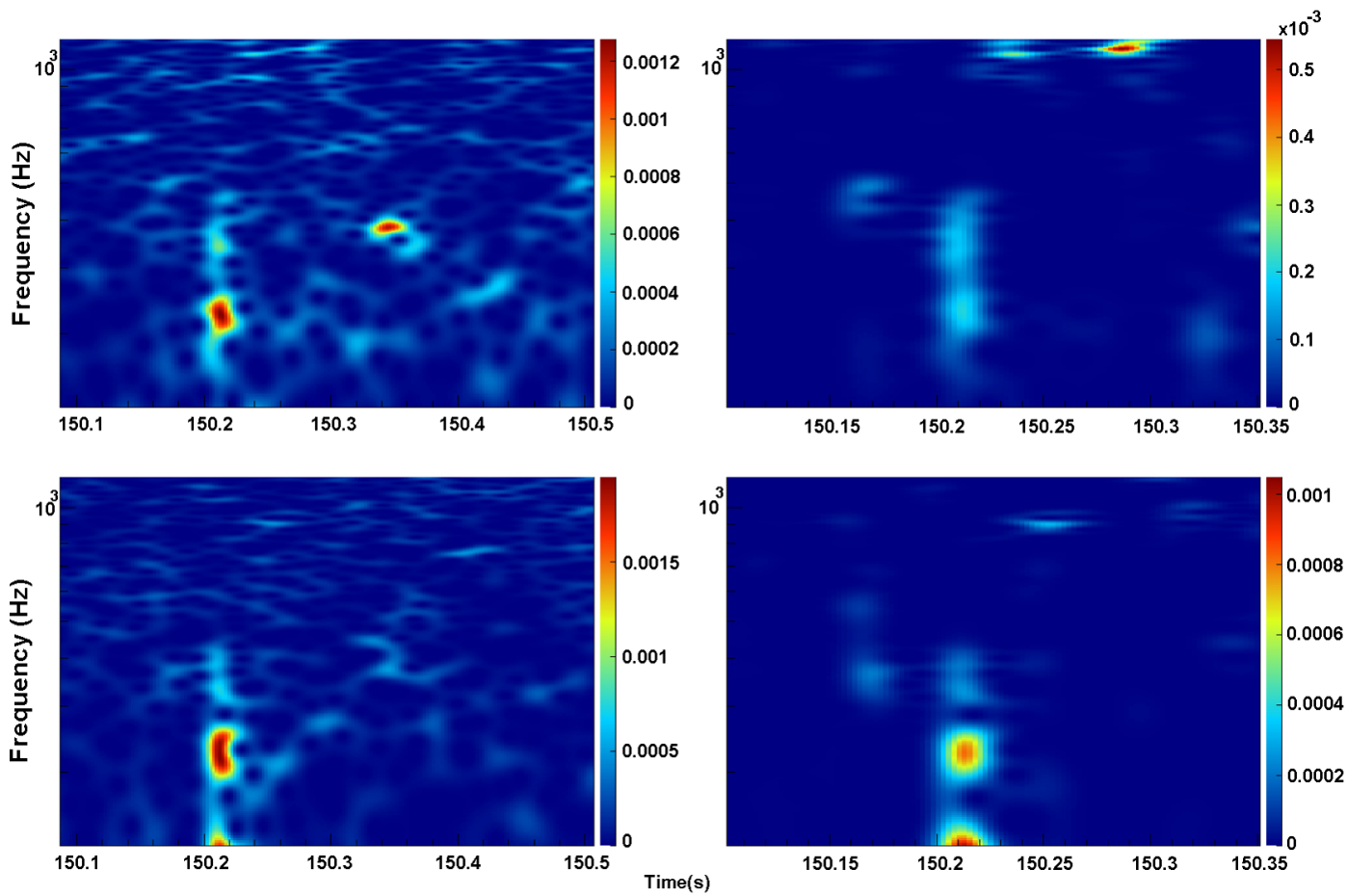

FIG. 25. This figure shows the spectrogram of signal detected at an output SNR of 10.6 by the cWB pipeline (left column, H1 at the top and L1 at the bottom); MuLaSECC pipeline (right column, H1 at the top and L1 at the bottom) detected an output SNR of 11.9. The injected Ott 2013 s11 signal was at SNR $=10$.
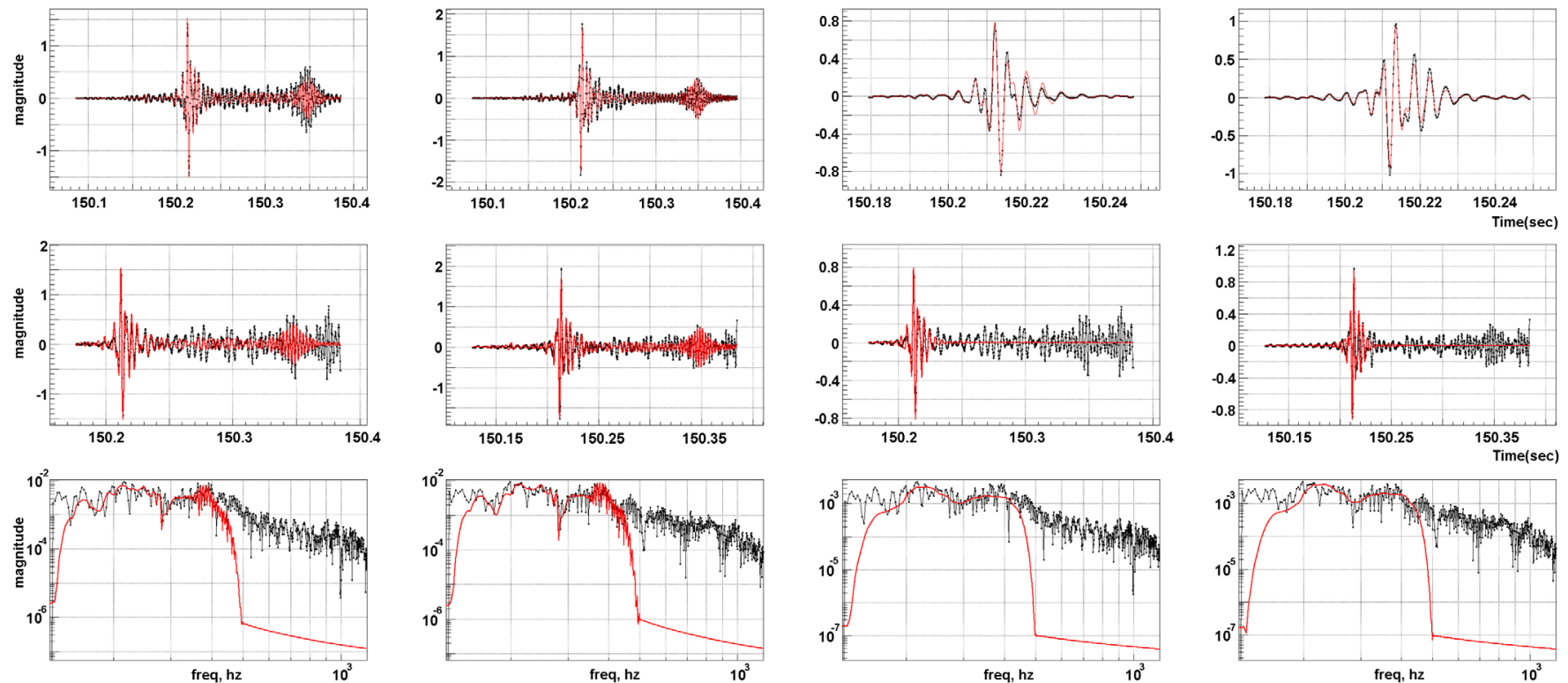

FIG. 26. The figure shows a comparative picture of the reconstruction of the detected signal waveform at the H1 and L1 detectors for Ott 2013 s11 waveforms as obtained by the cWB and the MuLaSECC pipelines for an injection with SNR 10. In this figure, the cWB results are in the two left hand columns and the MuLaSECC results are in the two right hand columns. For each pipeline, the left hand column presents results from $\mathrm{H} 1$ and the right hand column present results from L1. The top panels show the band-limited signal with the original in black and the reconstructed signal in red. The middle panels show the injected (in black) vs reconstructed (in red) whitened signal time series. The bottom panels display the amplitude spectra of the injected (black) vs reconstructed (red) whitened signal data. 


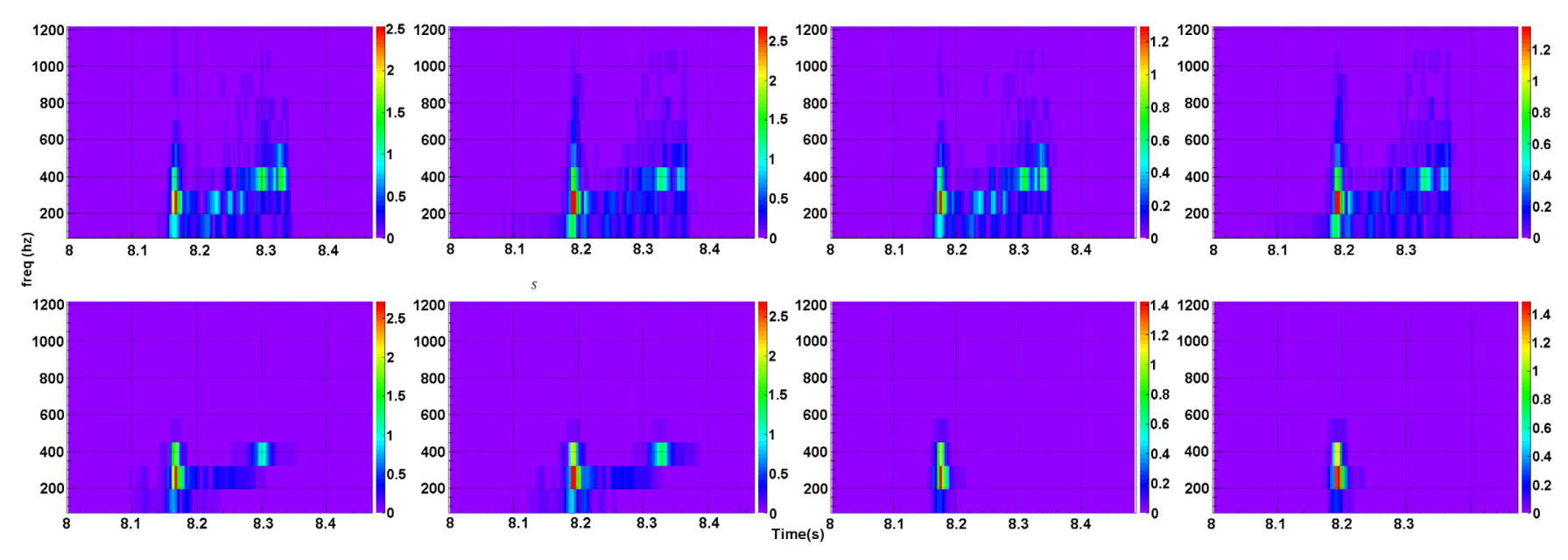

FIG. 27. The figure shows the reconstruction of the detected signal spectrum at the H1 and L1 detectors for Ott $2013 \mathrm{~s} 11$ waveforms as obtained by the cWB and the MuLaSECC pipelines for an injection with SNR 10. In this figure, the cWB results are in the two left hand columns and the MuLaSECC results are in the two right hand columns. For each pipeline, the left hand column presents results from H1 and the right hand column present results from L1. The top panels show the time-frequency plots for the band-limited signal. The bottom panels display the same for the reconstructed signal.
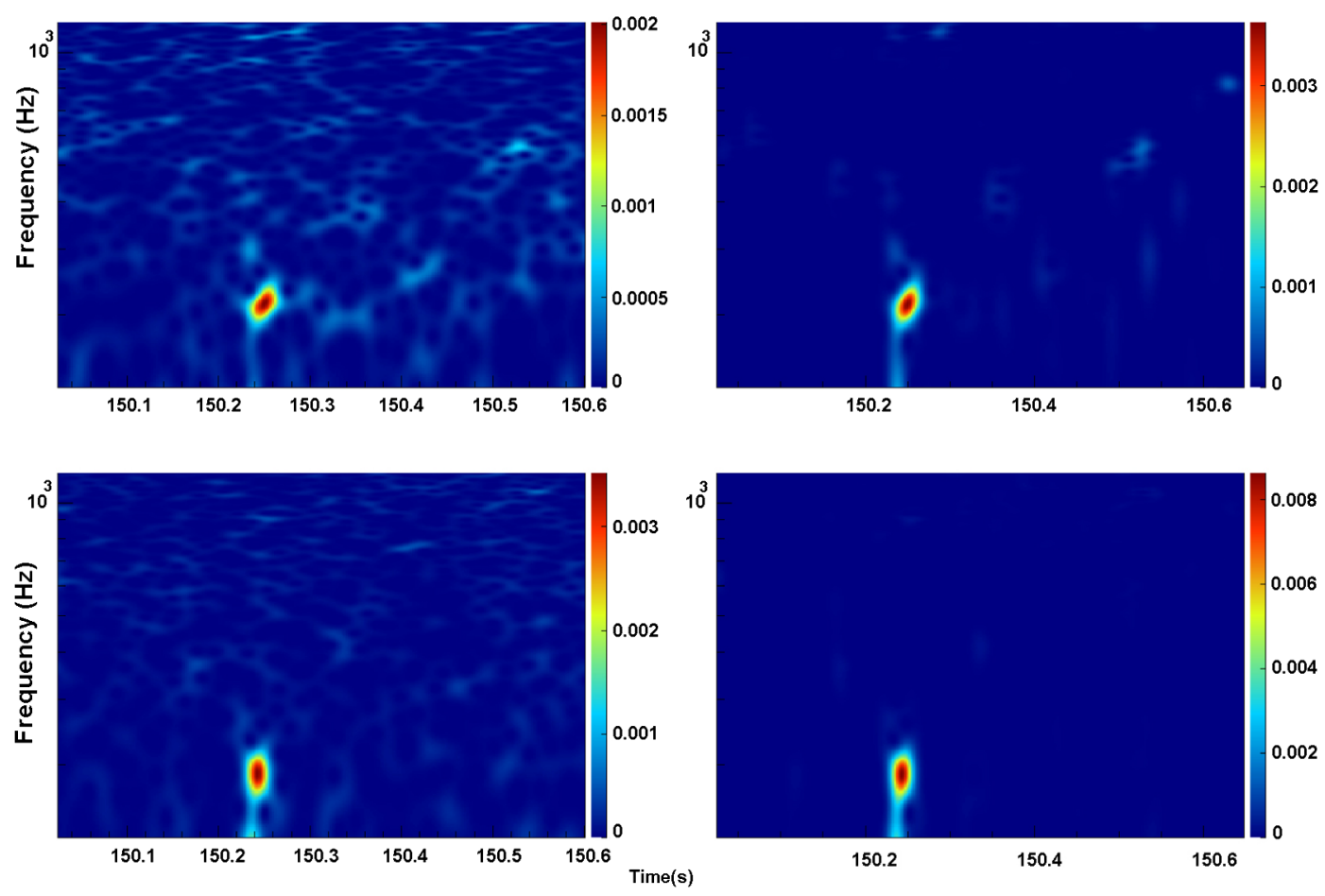

FIG. 28. This figure shows the spectrogram of signal detected at an output SNR of 14.7 by the cWB pipeline (left column, H1 at the top and L1 at the bottom); MuLaSECC pipeline (right column, H1 at the top and L1 at the bottom) detected an output SNR of 20.8. The injected Kuroda 2017 s11 signal was at $\mathrm{SNR}=15$. 

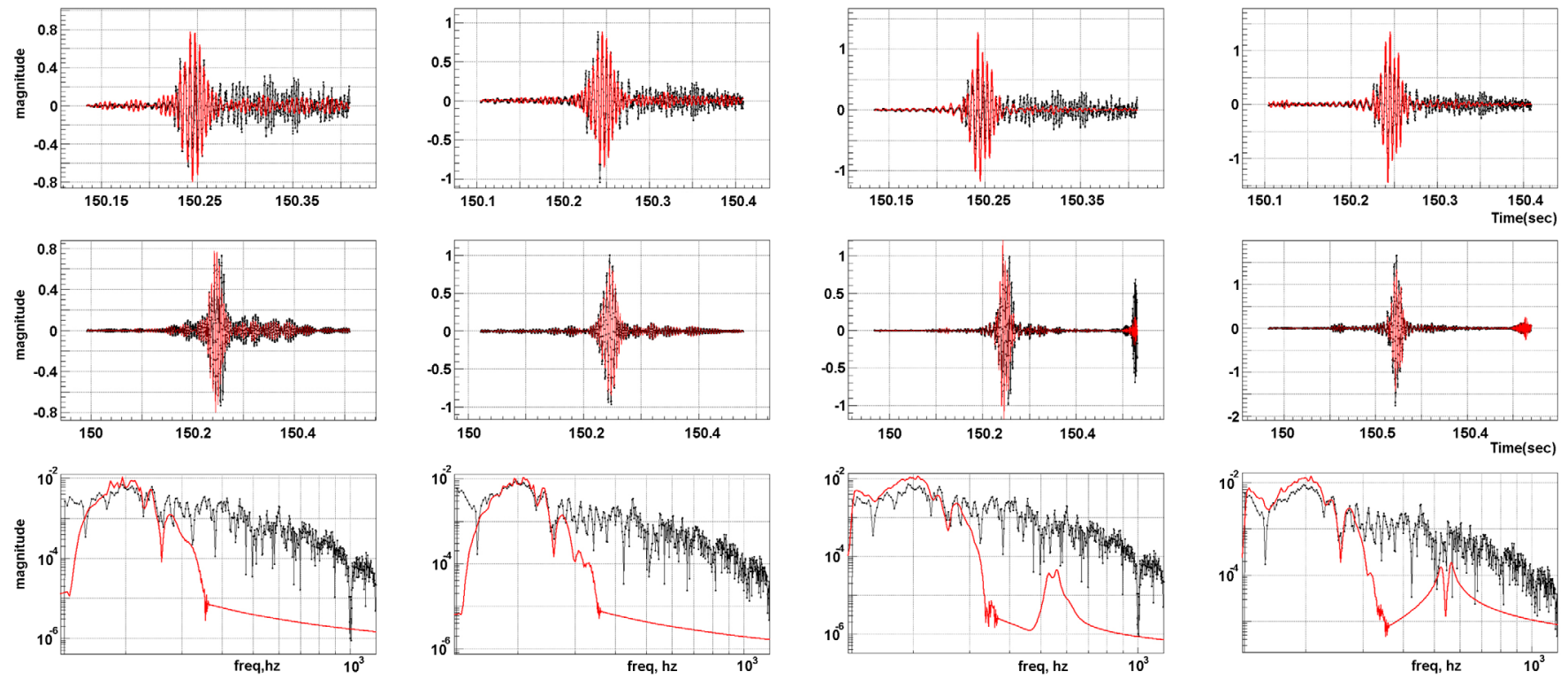

FIG. 29. The figure shows a comparative picture of the reconstruction of the detected signal waveform at the H1 and L1 detectors for Kuroda 2017 s11 waveforms as obtained by the cWB and the MuLaSECC pipelines for an injection with SNR 15. In this figure, the cWB results are in the two left hand columns and the MuLaSECC results are in the two right hand columns. For each pipeline, the left hand column presents results from $\mathrm{H} 1$ and the right hand column present results from L1. The top panels show the band-limited signal with the original in black and the reconstructed signal in red. The middle panels show the injected (in black) vs reconstructed (in red) whitened signal time series. The bottom panels display the amplitude spectra of the injected (black) vs reconstructed (red) whitened signal data.
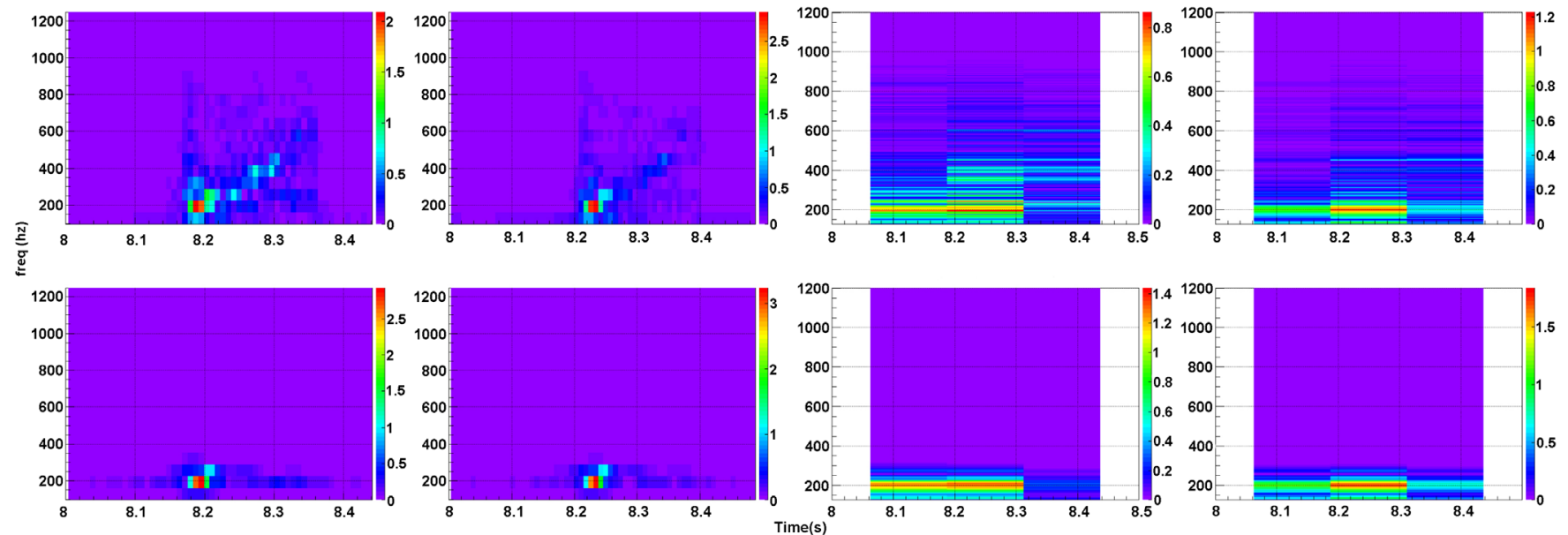

FIG. 30. The figure shows the reconstruction of the detected signal spectrum at the H1 and L1 detectors for Kuroda 2017 s11 waveforms as obtained by the cWB and the MuLaSECC pipelines for an injection with SNR 15. In this figure, the cWB results are in the two left hand columns and the MuLaSECC results are in the two right hand columns. For each pipeline, the left hand column presents results from $\mathrm{H} 1$ and the right hand column present results from L1. The top panels show the time-frequency plots for the band-limited signal. The bottom panels display the same for the reconstructed signal. 

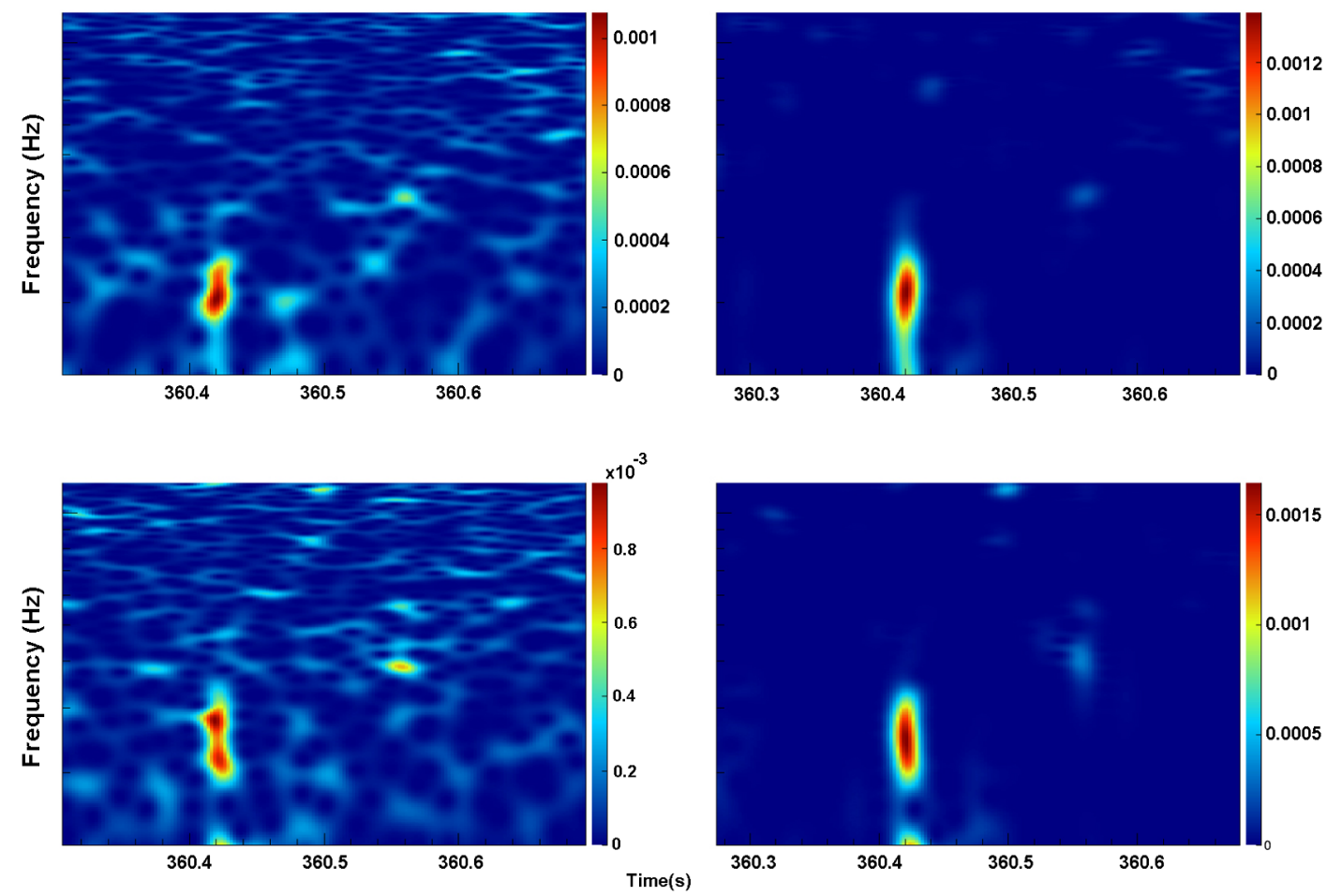

FIG. 31. This figure shows the spectrogram of signal detected at an output SNR of 14.7 by the cWB pipeline (left column, H1 at the top and L1 at the bottom); MuLaSECC pipeline (right column, H1 at the top and L1 at the bottom) detected an output SNR of 20.8. The injected Ott 2013 s27 signal was at $\mathrm{SNR}=15$.
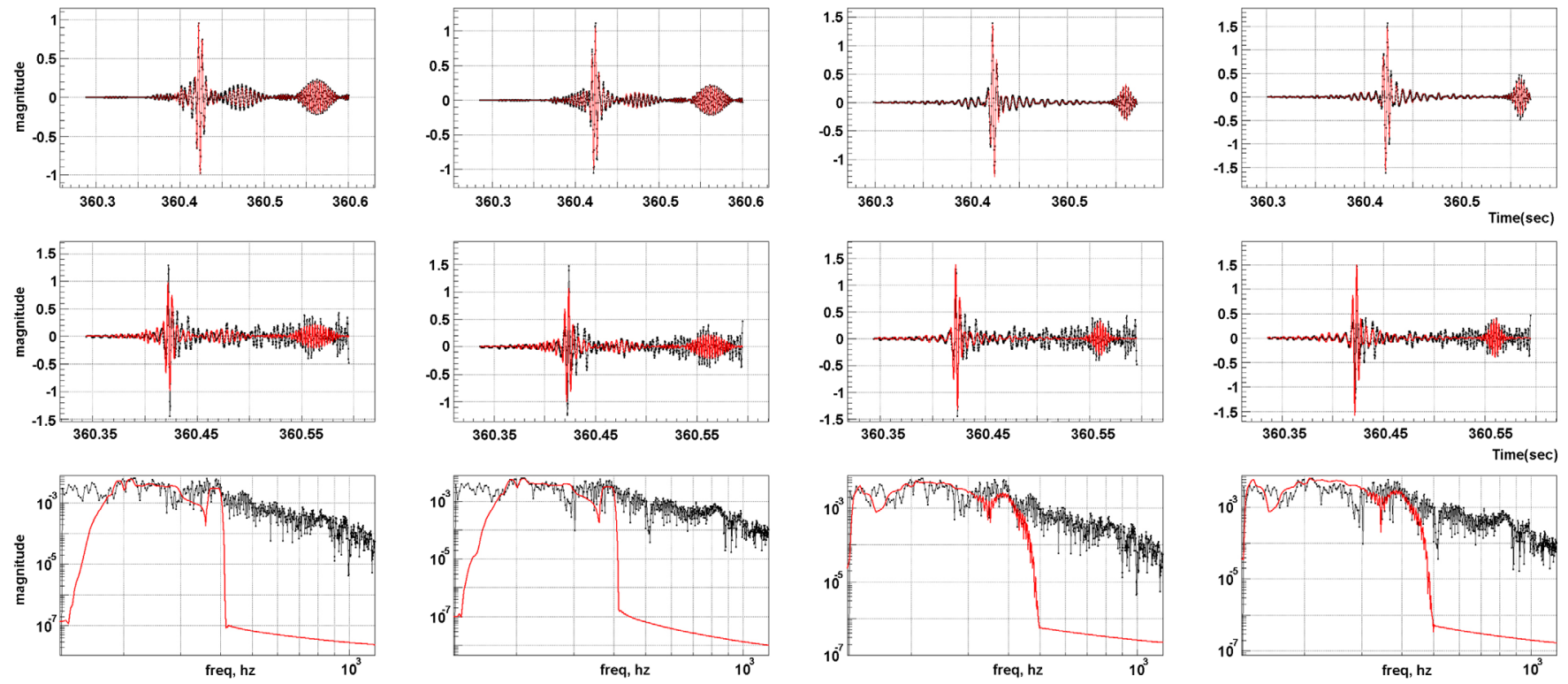

FIG. 32. The figure shows a comparative picture of the reconstruction of the detected signal waveform at the H1 and L1 detectors for Ott 2013 s27 waveforms as obtained by the cWB and the MuLaSECC pipelines for an injection with SNR 15. In this figure, the cWB results are in the two left hand columns and the MuLaSECC results are in the two right hand columns. For each pipeline, the left hand column presents results from $\mathrm{H} 1$ and the right hand column present results from L1. The top panels show the band-limited signal with the original in black and the reconstructed signal in red. The middle panels show the injected (in black) vs reconstructed (in red) whitened signal time series. The bottom panels display the amplitude spectra of the injected (black) vs reconstructed (red) whitened signal data. 

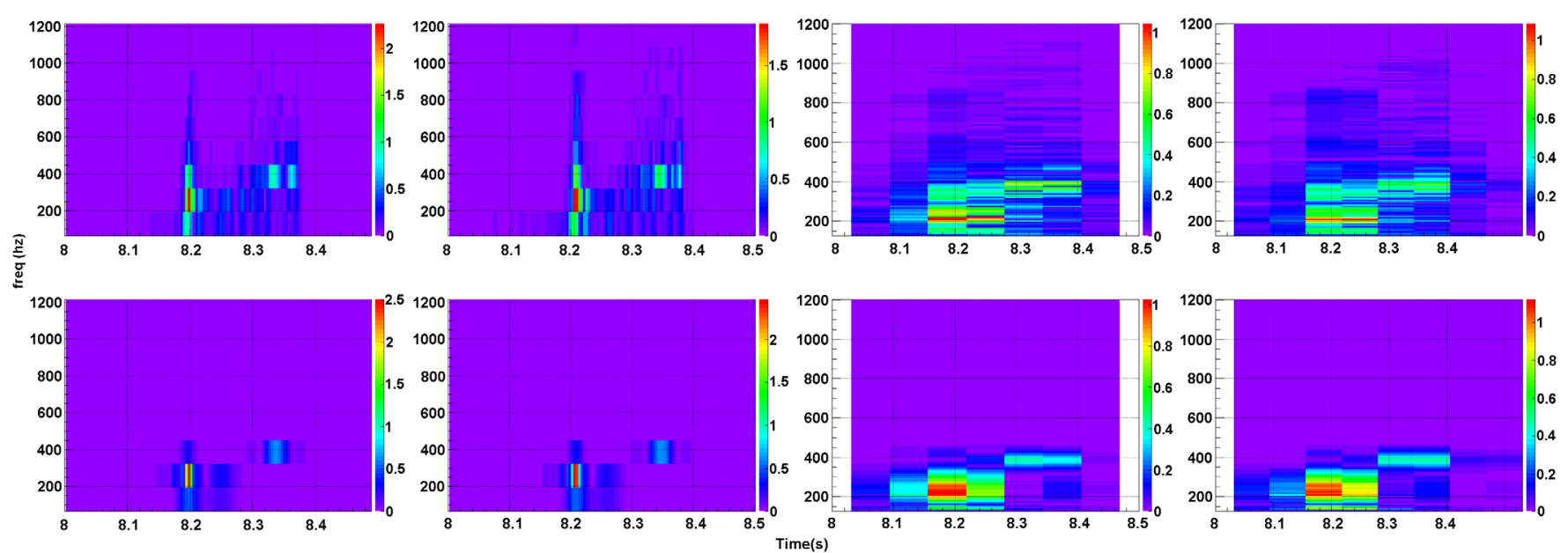

FIG. 33. The figure shows the reconstruction of the detected signal spectrum at the H1 and L1 detectors for Ott 2013 s 27 waveforms as obtained by the cWB and the MuLaSECC pipelines for an injection with SNR 15. In this figure, the cWB results are in the two left hand columns and the MuLaSECC results are in the two right hand columns. For each pipeline, the left hand column presents results from H1 and the right hand column present results from L1. The top panels show the time-frequency plots for the band-limited signal. The bottom panels display the same for the reconstructed signal.

\section{ACKNOWLEDGMENTS}

This work is supported by NSF Grants No. PHY1912630 (2019) and No. PHY-1505861 (2015) Use of LIGO's second observation run data is acknowledged. The authors gratefully acknowledge Dr. Marek Szczepanczyk (University of Florida, Gainesville) for his help with implementation of the cWB pipeline in the initial stages of this analysis in the context of GW search from CCSN sources. The authors are grateful for computational resources provided by the LIGO Laboratory and supported by NSF Grants No. PHY-0757058 and No. PHY0823459.
[1] B. Barish and R. Weiss, LIGO and the detection of gravitational waves, Phys. Today, no. 10, 52, 44 (1999).

[2] B. P. Abbott et al. (LIGO Scientific Collaboration), Observation of Gravitational Waves from a Binary Black Hole Merger, Phys. Rev. Lett. 116, 061102 (2016).

[3] T. Accadia et al., Virgo: A laser interferometer to detect gravitational waves, J. Instrum. 7, P03012 (2012).

[4] B. P. Abbott et al. (LIGO Scientific and Virgo Collaborations), Search for post-merger gravitational waves from the remnant of the binary neutron star merger GW170817, Astrophys. J. Lett. 851, L16 (2017).

[5] R. Abbott et al. (LIGO Scientific and Virgo Collaboration), GW190814: Gravitational waves from the coalescence of a 23 solar mass black hole with a 2.6 solar mass compact object, Astrophys. J. Lett. 896, L44 (2020).

[6] G. M. Harry (LIGO Scientific Collaboration), Advanced LIGO: The next generation of gravitational wave detectors, Classical Quant. Grav. 27, 084006 (2010).

[7] B. P. Abbott et al. (LIGO Scientific, Virgo, ASAS-SN, and DLT40 Collaborations), Optically targeted search for gravitational waves emitted by core-collapse supernovae during the first and second observing runs of Advanced LIGO and Advanced Virgo, Phys. Rev. D 101, 084002 (2020).
[8] S. D. Mohanty, M. Rakhmanov, S. Klimenko, and G. Mitselmakher, Variability of signal to noise ratio and likelihood analysis of gravitational wave burst signals using a network of detectors, Classical Quant. Grav. 23, 4799 (2006).

[9] M. Rakhmanov, Rank deciency of network matrix, Classical Quant. Grav. 23, S673 (2006).

[10] S. Klimenko, S. D. Mohanty, M. Rakhmanov, and G. Mitselmakher, Constraint likelihood analysis for a network of gravitational wave detectors, Phys. Rev. D 72, 122002 (2005).

[11] S. Mukherjee, L. Salazar, J. Mittelstaedt, and O. Valdez, Enhanced efficiency in detection of gravitational waves from supernovae using coherent network of detectors, Phys. Rev. D 96, 104033 (2017).

[12] C. Plapous, C. Marro, and P. Scalart, Improved signal-tonoise ratio estimation for speech enhancement, in IEEE Transactions on Audio, Speech, and Language Processing (IEEE, New York, 2006), Vol. 4, pp. 2098-2108, http://dx .doi.org/10.1109/TASL.2006.872621.

[13] P. Scalart and J. V. Filho, Speech enhancement based on a priori signal to noise estimation, in IEEE International Conference on Acoustics, Speech, and Signal Processing Conference Proceedings (IEEE, New York, 1996), Vol. 2, 
pp. 629-632, http://dx.doi.org/10.1109/ICASSP.1996 .543199.

[14] O. Cappe, Elimination of the musical noise phenomenon with the Ephraim and Malah noise suppressor, IEEE Trans. Speech Audio Process. 2, 345 (1994).

[15] Y. Ephram and D. Malah, Speech enhancement using a minimum mean-square error short-time spectral amplitude estimator, in IEEE Trans. Acoust., Speech, Signal Processing (IEEE, New York, 1984), Vol. 32, pp. 1109-1121, http:// dx.doi.org/10.1109/TASSP.1984.1164453.

[16] N. Kalchbrenner, E. Grefenstette, and P. Blunsom, A convolutional neural network for modelling sentences, arXiv:1404.2188.

[17] O. Abdel-Hamid, A. Mohamed, H. Jiang, and G. Penn, Applying convolutional neural networks concepts to hybrid NN-HMM model for speech recognition, in 2012 IEEE International Conference on Acoustics, Speech and Signal Processing (ICASSP), Kyoto, Japan (IEEE, New York, 2012, pp. 4277-4280, http://dx.doi.org/10.1109/ICASSP .2012.6288864.

[18] M. Liang and X. Hu, Recurrent convolutional neural network for object recognition, in Proceedings of the IEEE Conference on Computer Vision and Pattern Recognition, Boston (IEEE, New York, 2015), pp. 3367-3375, http://dx .doi.org/10.1109/CVPR.2015.7298958.

[19] S. Warren, Neural networks and statistical models, in Proceedings of the Nineteenth Annual SAS Users Group International Conference (SAS Institute Inc., Cary, NC, 1994).

[20] K. Fukushima, Neocognitron: A self-organizing neural network model for a mechanism of pattern recognition unaffected by shift in position, Biol. Cybern. 36, 193 (1980).

[21] S. Klimenko, I. Yakushin, A. Mercer, and G. Mitselmakher, Coherent method for detection of gravitational wave bursts, Classical Quant. Grav. 25, 114029 (2008).

[22] T. Kuroda, K. Kotake, and T. Takiwaki, A new gravitationalwave signature from standing accretion shock instability in supernovae, Astrophys. J. Lett. 829, L14 (2016).

[23] T. Kuroda, K. Kotake, K. Hayama, and T. Takiwaki, Correlated signatures of gravitational-wave and neutrino emission in three-dimensional general-relativistic corecollapse supernova simulations, Astrophys. J. 851, 62 (2017).

[24] C. D. Ott, E. Abdikamalov, P. Moesta, R. Haas, S. Drasco, E. P. O'Connor, C. Reisswig, C. A. Meakin, and E. Schnetter, General-relativistic simulations of three-dimensional corecollapse supernovae, Astrophys. J. 768, 115 (2013).

[25] T. Akutsu, M. Ando, K. Arai et al., KAGRA: 2.5 generation interferometric gravitational wave detector, Nat. Astron. 3, 35 (2019).

[26] https://stellarcollapse.org/.

[27] A. Mezzacappa, P. Marronetti, R. E. Landfield et al., Gravitational-wave signal of a core-collapse supernova explosion of a 15M star, Phys. Rev. D 102, 023027 (2020).

[28] S. W. Bruenn, J. M. Blondin, W. R. Hix et al., Chimera: A massively parallel code for core-collapse supernova simulation, Astrophys. J. Suppl. Ser. 248, 11 (2020).

[29] J. Powell and B. Muller, Three-dimensional core-collapse supernova simulations of massive and rotating progenitors, Mon. Not. R. Astron. Soc. 494, 4665 (2020).
[30] B. Mueller, H. Janka, and H. Dimmelmeier, A new multidimensional general relativistic neutrino hydrodynamic code for core-collapse supernovae. I. method and code tests in spherical symmetry, Astrophys. J. Suppl. Ser. 189, 104 (2010).

[31] S. Hild, S. Chelkowski, and A. Freise, Pushing towards the ET sensitivity using "conventional" technology, arXiv:0810 .0604 .

[32] www.ligo.org.

[33] J. W. Murphy, C. D. Ott, and A. Burrows, A model for gravitational wave emission from neutrino-driven corecollapse supernovae, Astrophys. J. 707, 1173 (2009).

[34] B. Muller, H. T. Janka, and A. Marek, A new multidimensional general relativistic neutrino hydrodynamics code of core-collapse supernovae. III. Gravitational wave signals from supernova explosion models, Astrophys. J. 766, 43 (2013).

[35] H. Andresen, B. Mueller, E. Mueller, and H. T. Janka, Gravitational wave signals from 3D neutrino hydrodynamics simulations of core-collapse supernovae, Mon. Not. R. Astron. Soc. 468, 2032 (2017).

[36] B. P. Abbott et al. (LIGO Scientific and Virgo Collaborations), First targeted search for gravitational-wave bursts from core-collapse supernovae in data of first-generation laser interferometer detectors, Phys. Rev. D 94, 102001 (2016).

[37] J. Hough and S. Rowan, Laser interferometry for the detection of gravitational waves, J. Opt. A 7, S257 (2005).

[38] K. Hayama, T. Kuroda, K. Kotake, and T. Takiwaki, Circular polarization of gravitational waves from non-rotating supernova cores: A new probe into the pre-explosion hydrodynamics, Mon. Not. R. Astron. Soc. 477, L96 (2018).

[39] S. E. Gossan, P. Sutton, A. Stuver, M. Zanolin, K. Gill, and C. D. Ott, Observing gravitational waves from core-collapse supernovae in the advanced detector era, Phys. Rev. D 93, 042002 (2016).

[40] D. Lai, in AIP Conf. Proc. 575: Astrophysical Sources for Ground-Based Gravitational Wave Detectors, edited by J. M. Centrella (American Institute of Physics, Melville, 2001), p. 246, http://www.gbv.de/dms/goettingen/ 332431231.pdf.

[41] C. D. Ott, A. Burrows, T. A. Thompson, E. Livne, and R. Walder, The spin periods and rotational profiles of neutron stars at birth, Astrophys. J. Suppl. Ser. 164, 130 (2006).

[42] M. A. Bizouard, P. Maturana-Russel, A. Torres-Forn, M. Obergaulinger, P. Cerd-Durn, N. Christensen, J. A. Font, and R. Meyer, Inference of proto-neutron star properties from gravitational-wave data in core-collapse supernovae, Phys. Rev. D 103, 063006 (2021).

[43] M. Cavaglia, S. Gaudio, T. Hansen, K. Staats, M. Szczepanczyk, and M. Zanolin, Improving the background of gravitational-wave searches for core collapse supernovae: A machine learning approach, Mach. Learn. Sci. Technol. 1, 015005 (2020).

[44] A. Iess, E. Cuoco, F. Morawski, and J. Powell, Corecollapse supernova gravitational-wave search and deep learning classification, arXiv:2001.00279 [Mach. Learn. Sci. Technol. (to be published)].

[45] M. L. Chan, I. S. Heng, and C. Messenger, Detection and classification of supernova gravitational wave 
signals: A deep learning approach, Phys. Rev. D 102, 043022 (2020).

[46] D. George, H. Shen, and E. A. Huerta, Glitch classification and clustering for LIGO with deep transfer learning, Phys. Rev. D 97, 101501 (2018).

[47] P. Astone, P. Cerdá-Durán, I. Di Palma, M. Drago, F. Muciaccia, C. Palomba, and F. Ricci, New method to observe gravitational waves emitted by core collapse supernovae, Phys. Rev. D 98, 122002 (2018).

[48] M. Lopez Portilla, I. Di Palma, M. Drago, P. Cerda-Duran, and F. Ricci, Deep learning for multimessenger core-collapse supernova detection, Phys. Rev. D 103, 063011 (2021).

[49] D. Ciresan, U. Meier, J. Masci et al., Flexible high performance convolutional neural networks for image classification, Proceedings of the Twenty-Second International Joint Conference on Artificial Intelligence (2011), Vol. 2, pp. 1237-1242.

[50] I. Goodfellow, Y. Bengio, and A. Courville, Deep Learning (MIT Press, Cambridge, MA, 2016).

[51] J. Wu, Introduction to convolutional neural networks, https://www.semanticscholar.org/ (2017).

[52] K. Murphy, Machine Learning: A Probabilistic Perspective (MIT, Cambridge, MA, 2012).
[53] A. Gelman and J. Hill, Data Analysis Using Regression and Multilevel/Hierarchical Models (Cambridge University Press, Cambridge, England, 2006).

[54] C. K. Enders, Applied Missing Data Analysis (Guilford Press, New York, 2010).

[55] G. KaltonD. Kasprzyk, The treatment of missing survey data, Survey Methodol. 12, 1 (1986).

[56] D. Rubin, Multiple Imputation for Nonresponse in Surveys, Wiley Series in Probability and Statistics (Wiley, New York, 1987).

[57] Y. C. Yuan, Multiple Imputation for Missing Data: Concepts and New Development (SAS Institute Inc., Rockville, MD, 2010), Vol. 49, p. 111.

[58] S. Van Buuren, 2. Multiple Imputation: Flexible Imputation of Missing Data, Chapman and Hall/CRC Interdisciplinary Statistics Series 20125245 (Chapman and Hall/CRC, London, 2012).

[59] https://icecube.wisc.edu/.

[60] http://www-sk.icrr.u-tokyo.ac.jp/sk/index-e.html.

[61] S. D. Mohanty and E. Fahnestock, Adaptive spline fitting with particle swarm optimization, Comput. Stat. https:// doi.org/10.1007/s00180-020-01022-x (2020). 